\title{
Introduction to Data Visualization
}

\author{
Dario Rodighiero \\ École polytechnique fédérale de Lausanne (EPFL)
}

Institut National d'Histoire de l'Art, 3 October 2018

https://dariorodighiero.com 


\section{1.}

Charts, Diagrams, Infographics, Maps, Visualizations, and Data Visualizations 


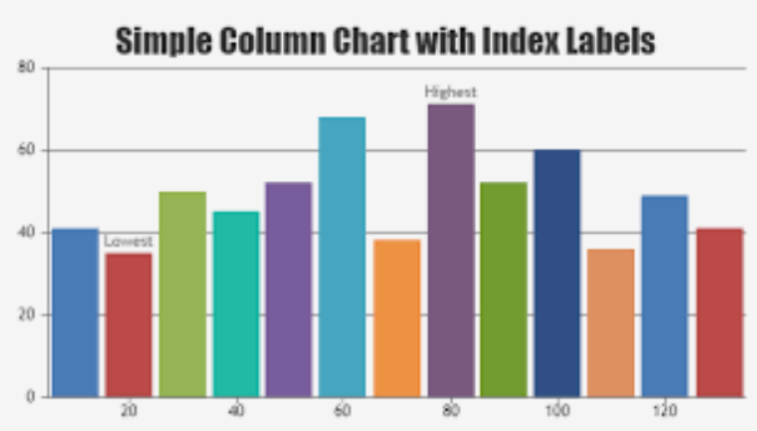

Beautiful PHP Charts \& Graphs | CanvasJS canvasjs.com

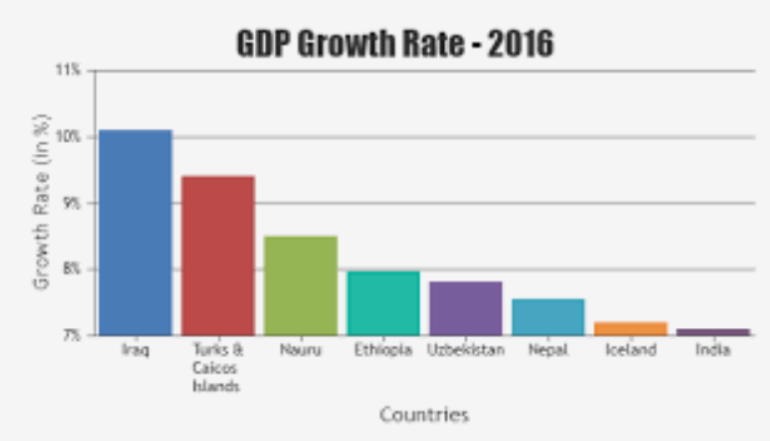

jQuery Charts \& Graphs | CanvasJS

canvasjs.com

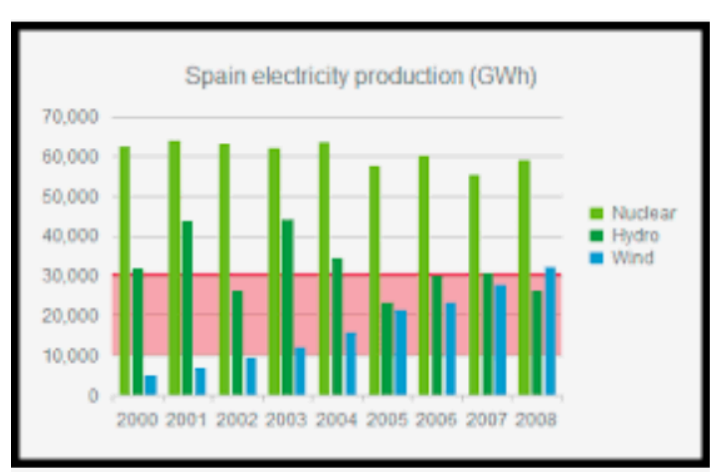

Charts Control - Kendo UI with support

telerik.com

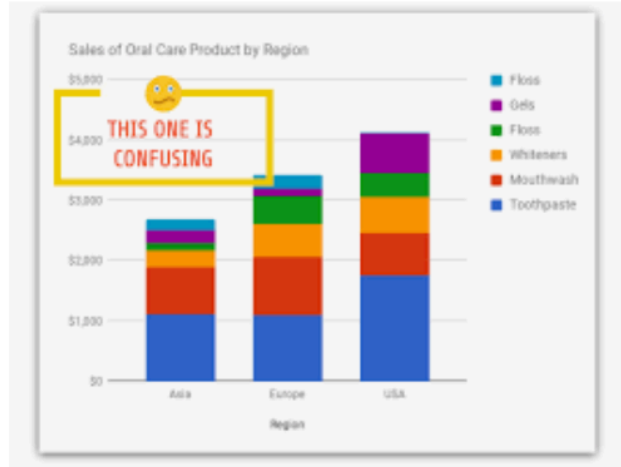

Eye-Catching Sales Charts - Venngage venngage.com

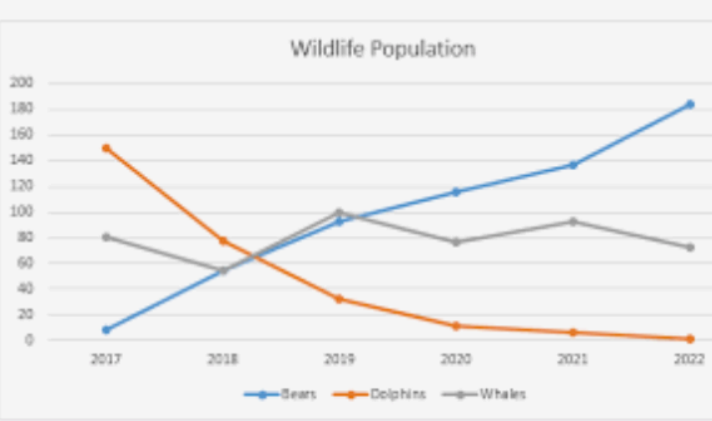

Line Chart in Excel - Easy Excel Tutorial excel-easy.com

Composition of 38th Parliament
of Canada as of May 19, 2005

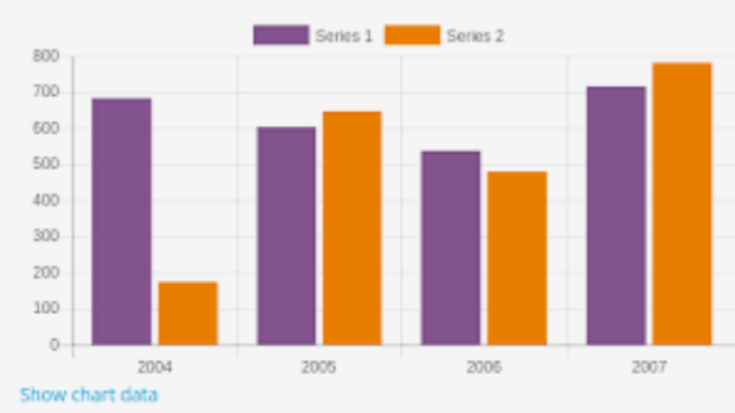

Charts API - MoodleDocs

docs.moodle.org

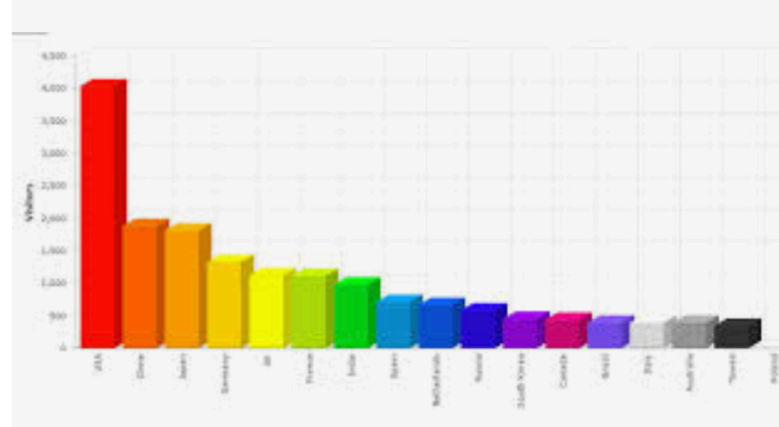

3D Column Chart - amCharts

amcharts.com

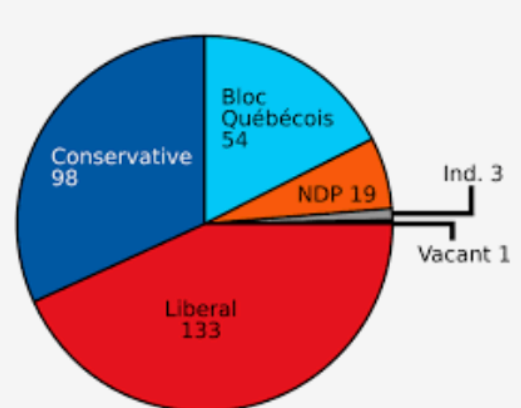

Chart - Wikipedia

en.wikipedia.org

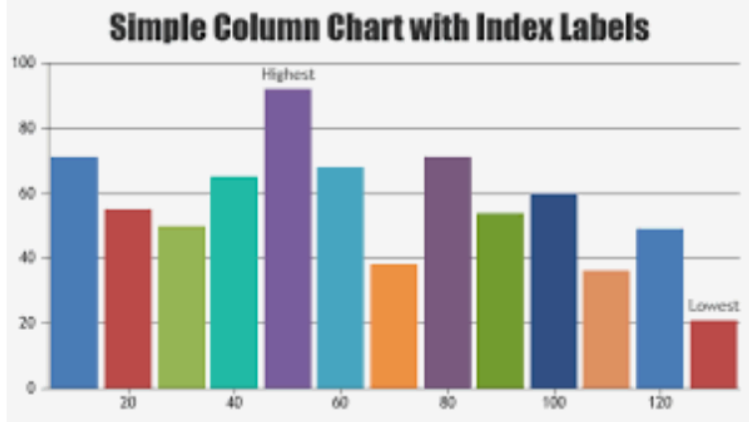

Beautiful JavaScript Charts \& Graphs .. canvasjs.com

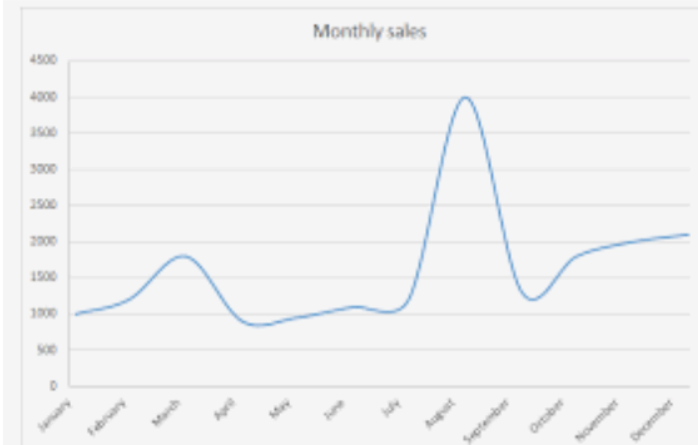

stop those lying line charts - Signal m.signalvnoise.com

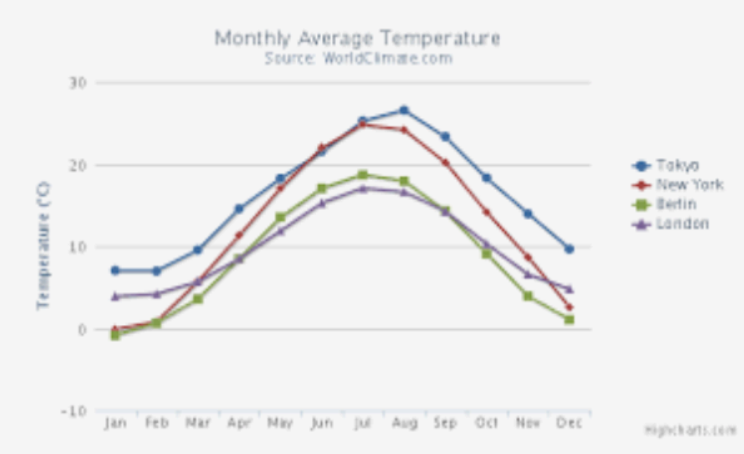

Line chart | Highcharts

highcharts.com

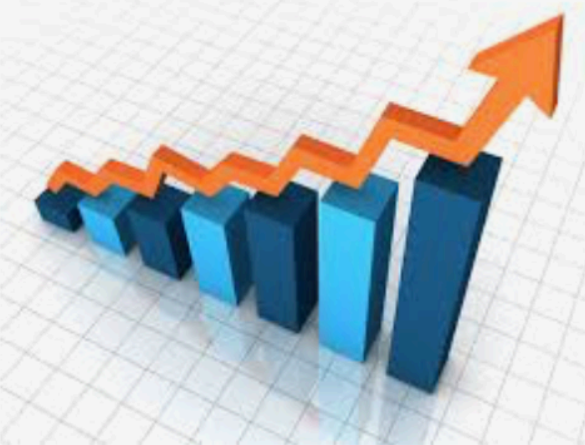

Create Interactive Charts with ui-grid .. algotech.solutions

Google Search for "Charts" 


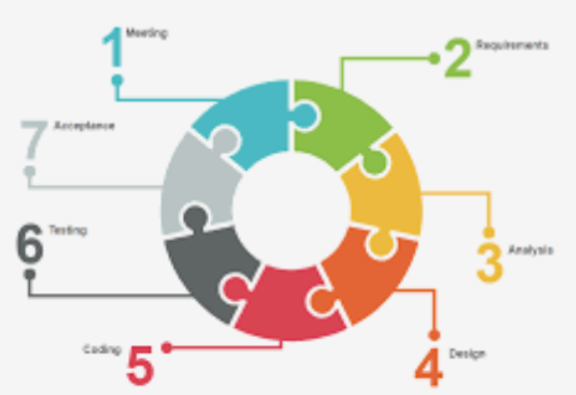

SmartDraw - Create Flowcharts, Floor ... smartdraw.com

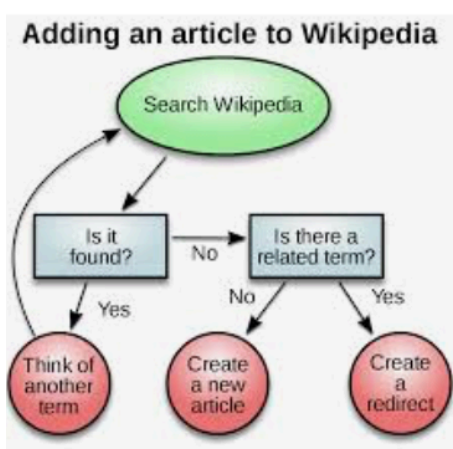

Diagram - Wikipedia en.wikipedia.org

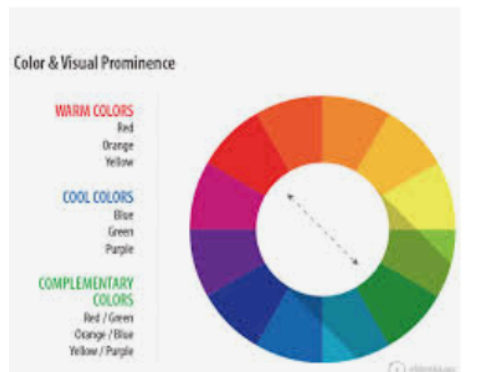

Diagrams That Explain Conte. orbitmedia.com

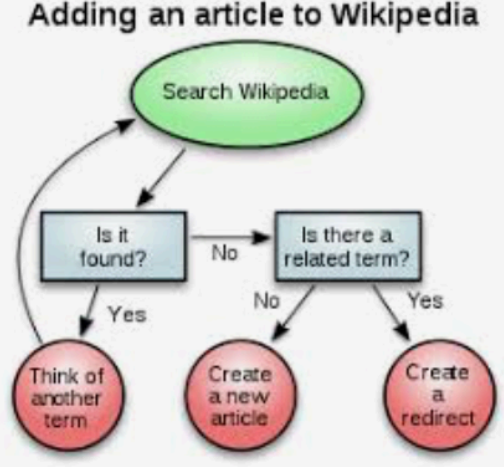

Diagram - Wikipedia en.wikipedia.org

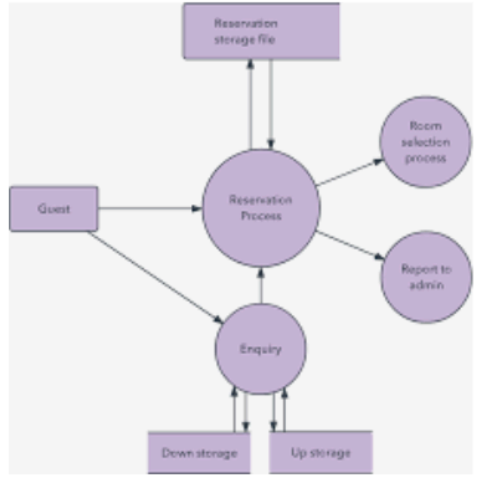

What is a Data Flow Diagram ... lucidchart.com

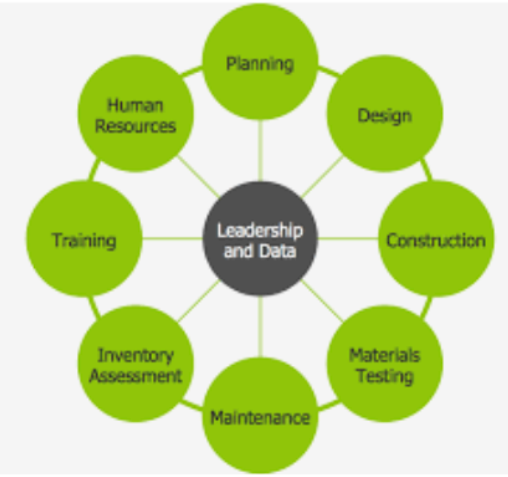

Circle Diagrams conceptdraw.com

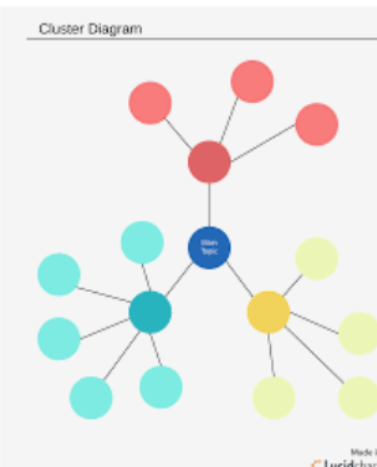

What Is a Cluster Diagram lucidchart.com

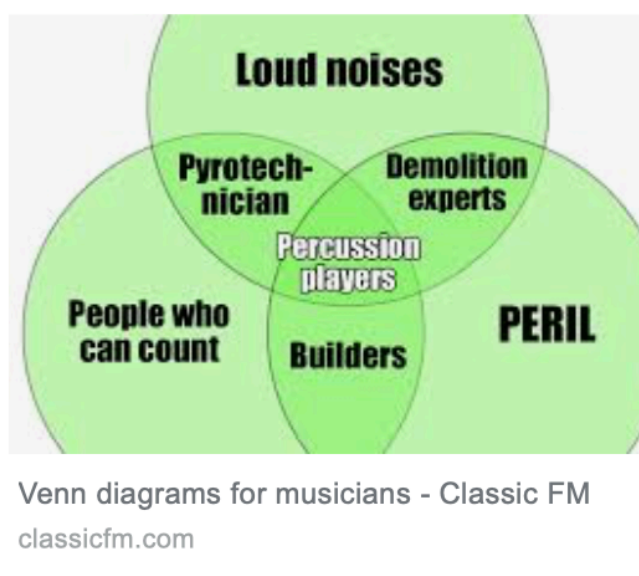

Create UML diagrams onli... SmartDraw Diagrams smartdraw.com

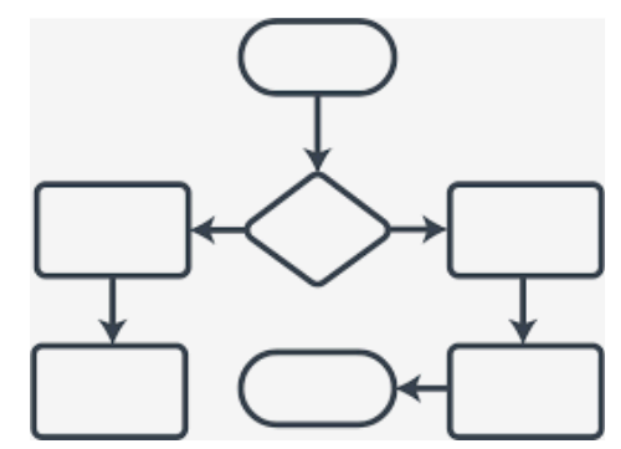

All About Business Process Mapping lucidchart.com

Google Search for "Diagrams" 


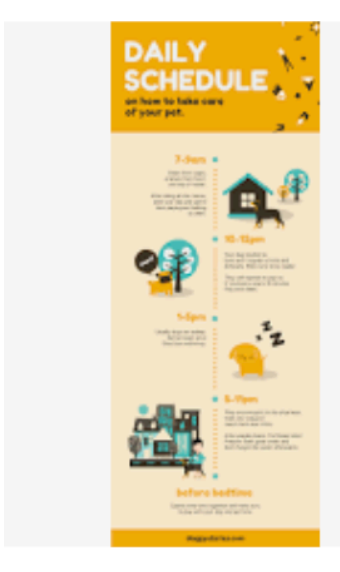

Free Online Infographic $\mathrm{M}$ canva.com

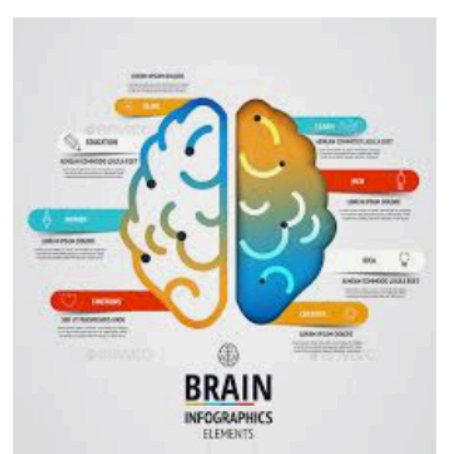

Creative Brain Infographic.. graphicriver.net

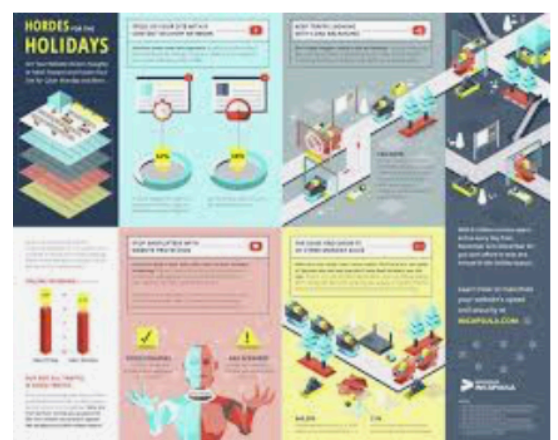

The 45 Most Creative Infographics ... columnfivemedia.com

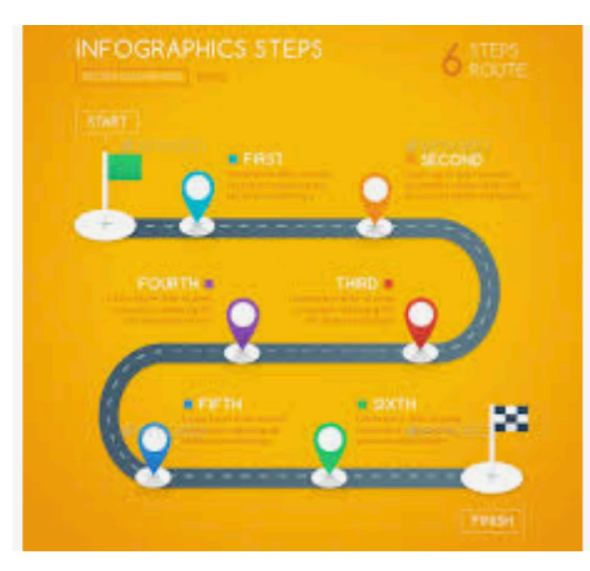

Milestone Infographics - 6 Steps by graphicriver.net

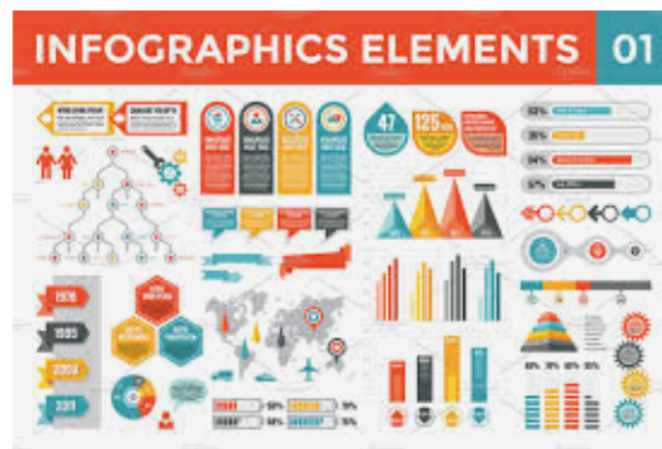

Infographics Elements $01 \sim$ Presentation ... creativemarket.com

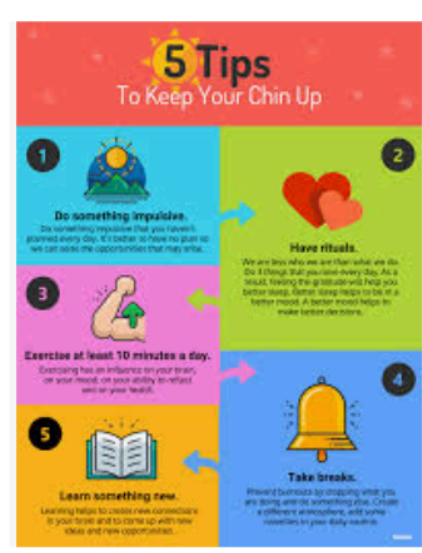

Brochures, Flyers \& More -.. venngage.corn

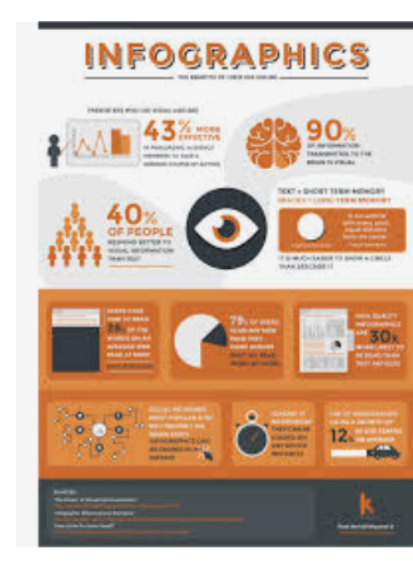

Visual.ly

visual.

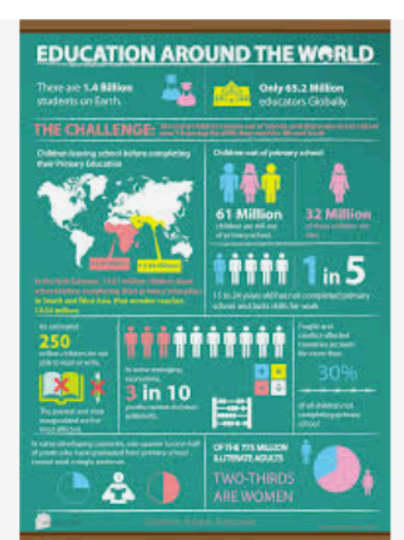

10 Great Infographics for E. piktochart.com

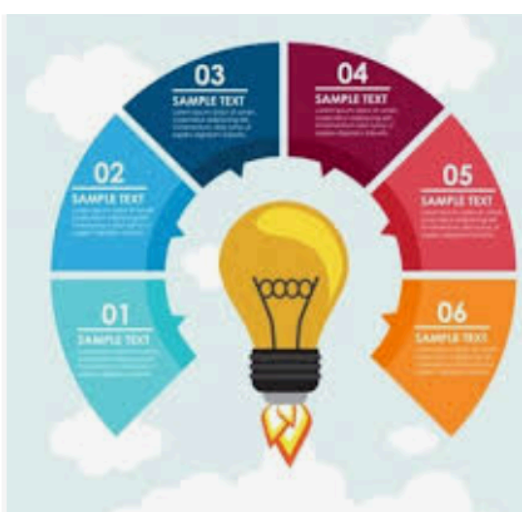

Infographics Vectors, Photos and P. reepik.com

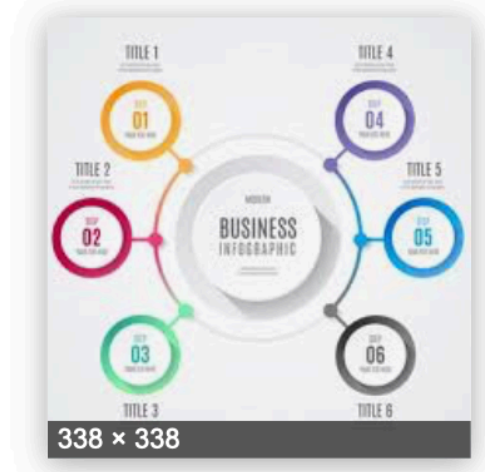

Infographics Vectors, Phot.. freepik.com

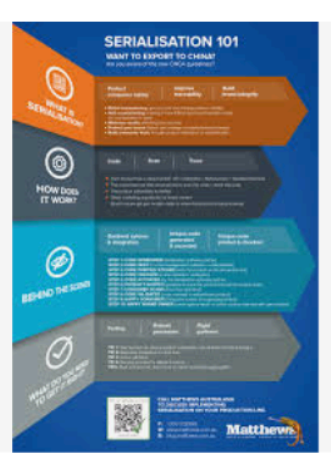

manufacturing industry matthews.com.au

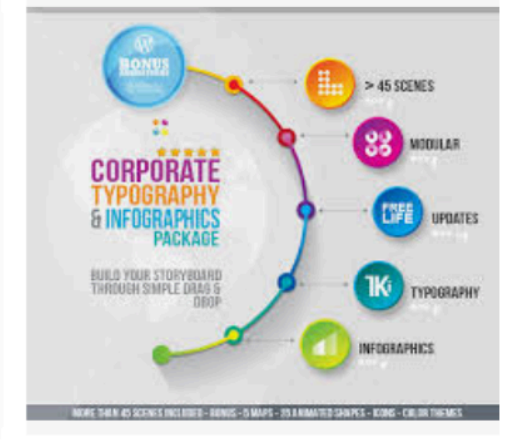

Corporate Typography \& Infog... videohive.net

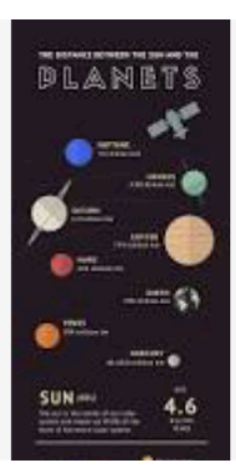

Free Online Infographic M... canva.com

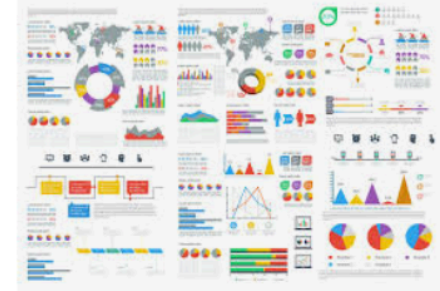

Infographics template IIlu creativemarket.com

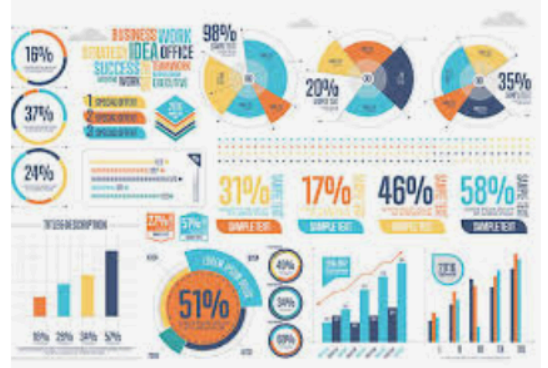

Infographics Clip Art, Vector Image.. istockphoto.com

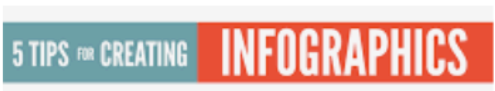

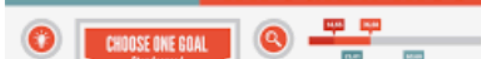

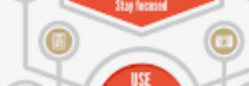

(4)

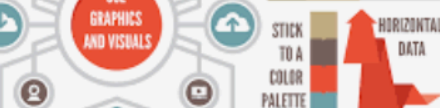

() BO\%

5 Tips for Creating Infographics digitalchalk.com

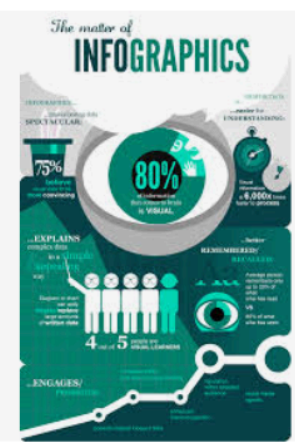

Our Infographic Design Se... quintagroup.com

Google Search for "Infographics" 


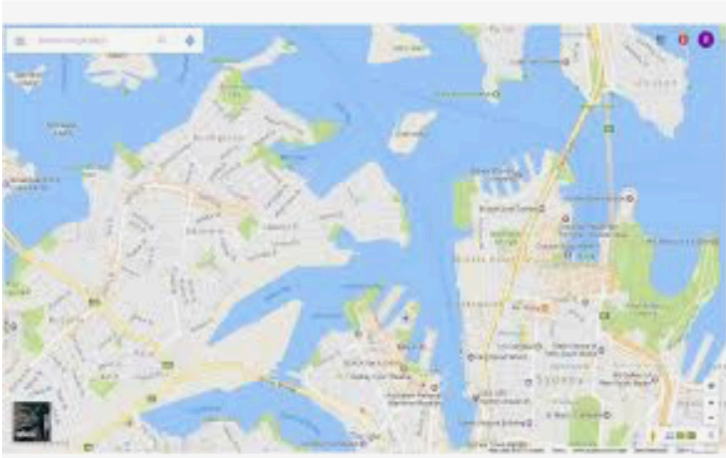

Developer Guide | Maps URLs | Google ...

developers.google.com

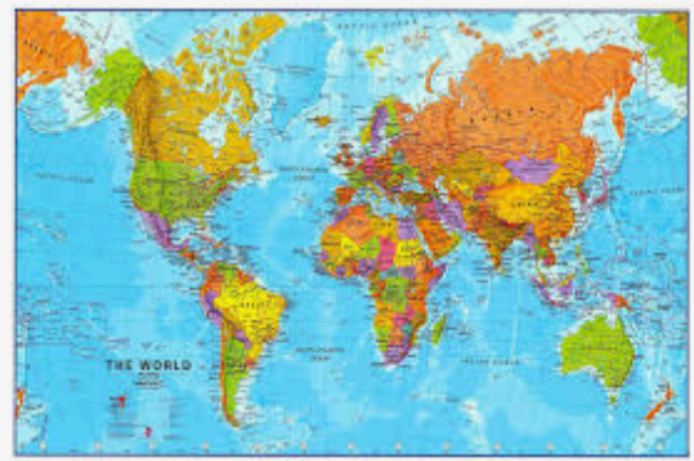

International 1:20 million Supermap

mapworld.com.au

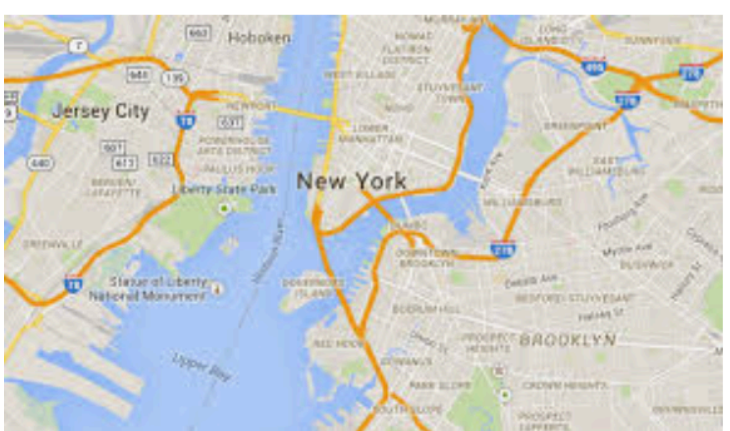

Snazzy Maps - Free Styles for Google Maps snazzymaps.com

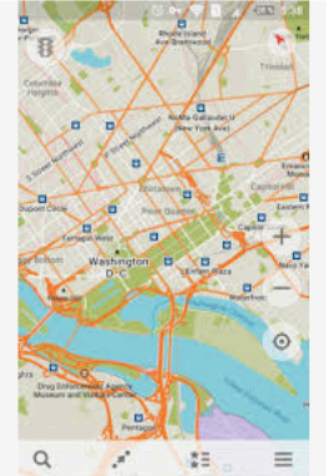

Maps.me - Wikipedia

en.wikipedia.ors

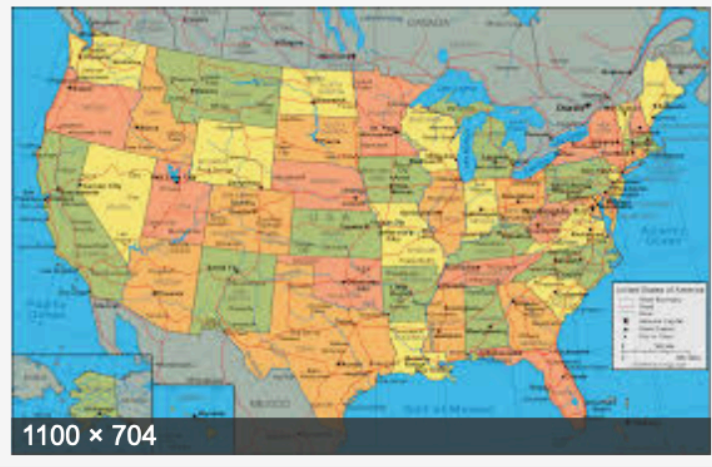

United States Map and Satellite Image

$$
\text { geology.com }
$$

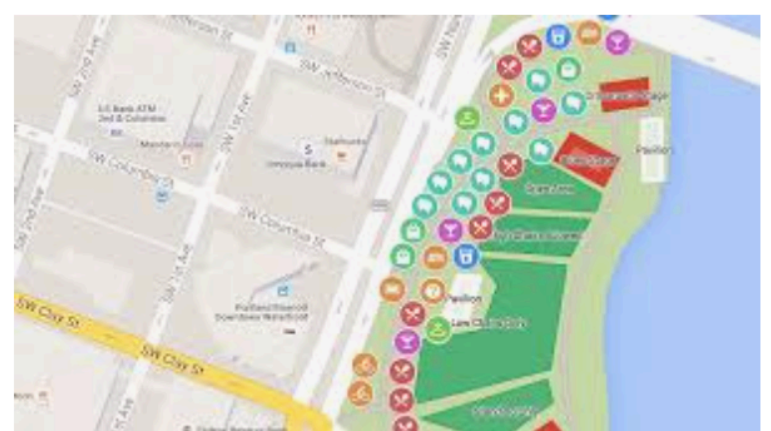

My Maps - About - Google Maps google.com

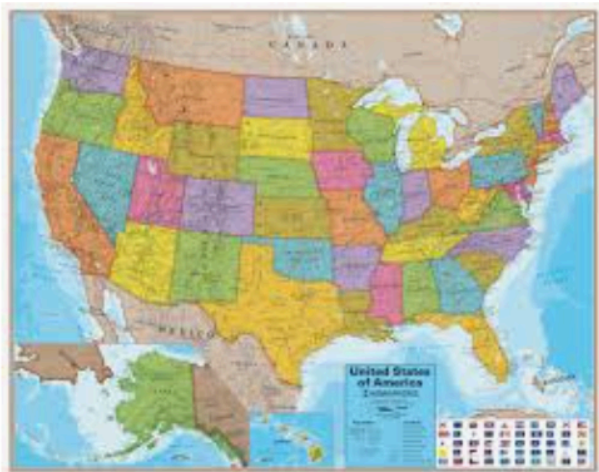

Wall Map of the United States

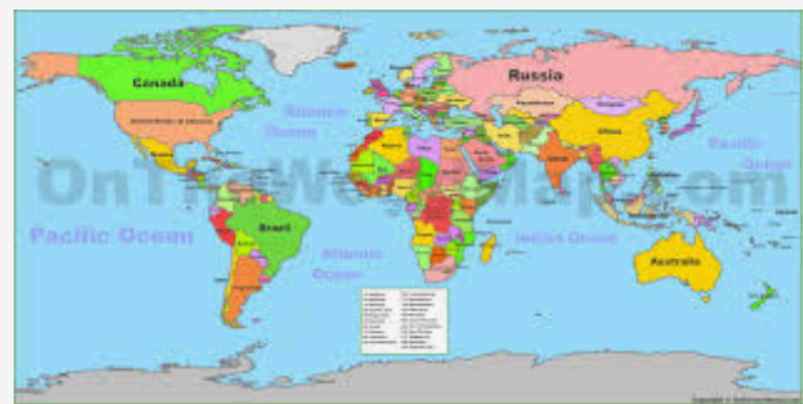

World Maps | Maps of all countries ontheworldmap.com

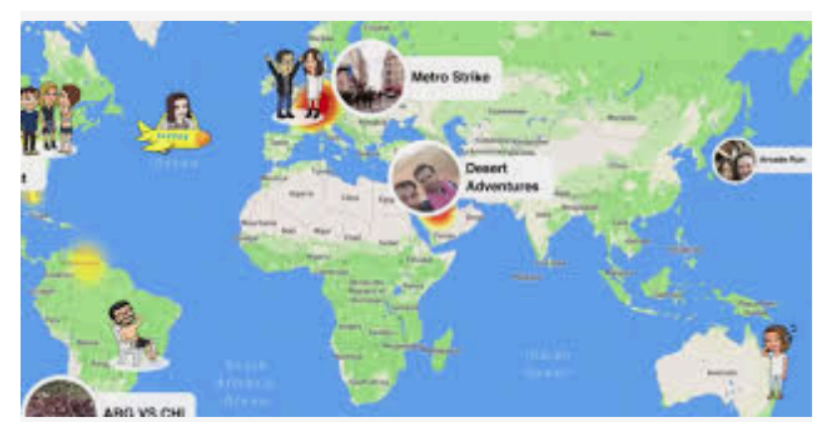

Explore feature makes Maps a social thenextweb.com

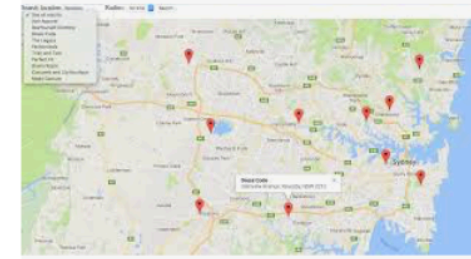

Creating a Store Locator on

developers.google.com

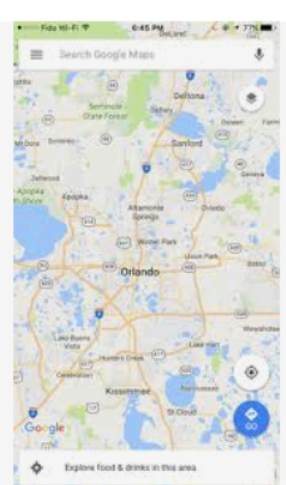

Google Maps (app) - Wikipedia en.wikipedia.org

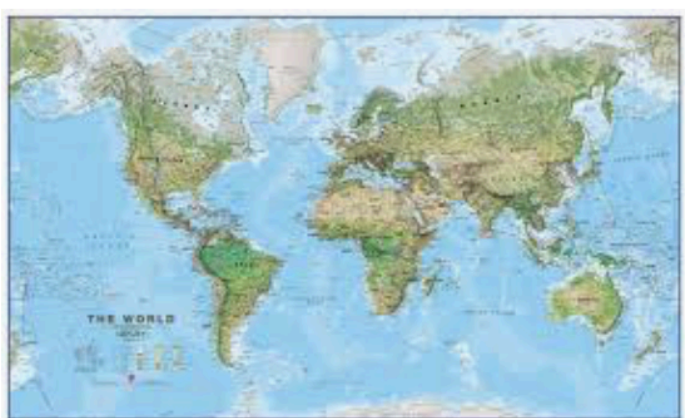

World Wall Map Environmenta mapsinternational.co.uk

\section{Google Search for "Maps"}




\section{ill o, 098 = What Makes A Good Visualization?

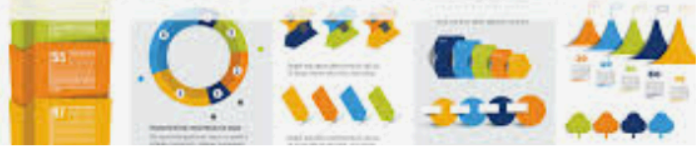

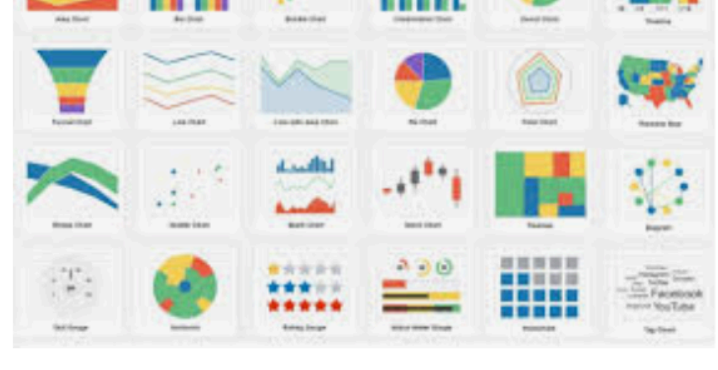

What makes a good visualization? | E .. e-nor.com
Latest Version of OBIEE 12C further Latest Version of

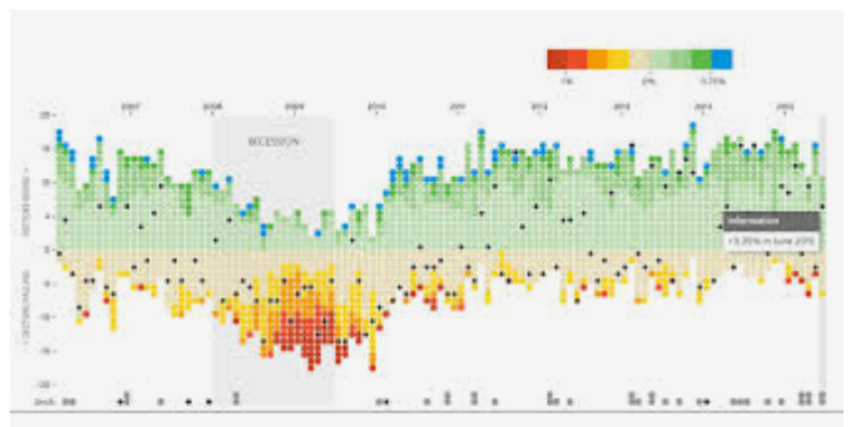

The Best Topical Data Visualizations of

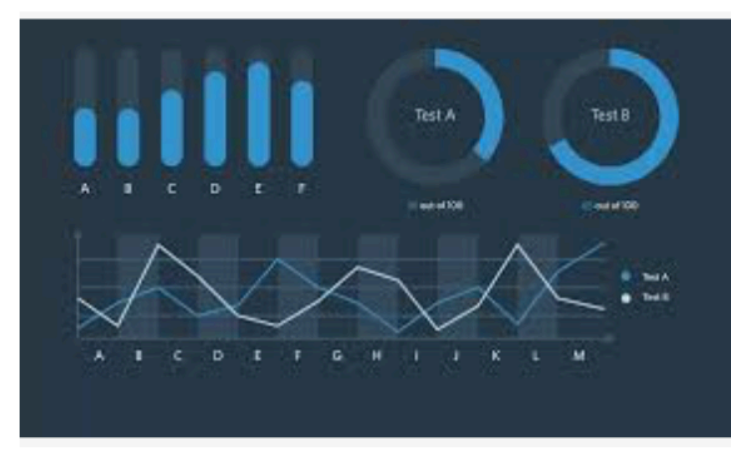

Getting it right: why infographics are blog.prototypr.io

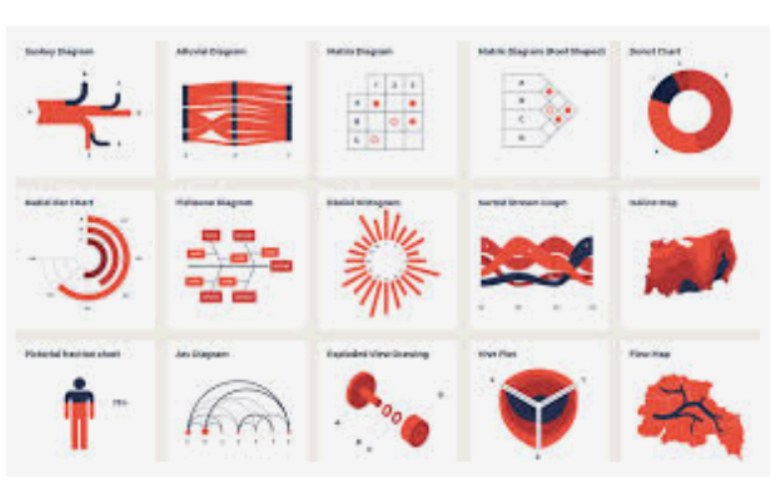

Data Visualizations

datainnovation.org

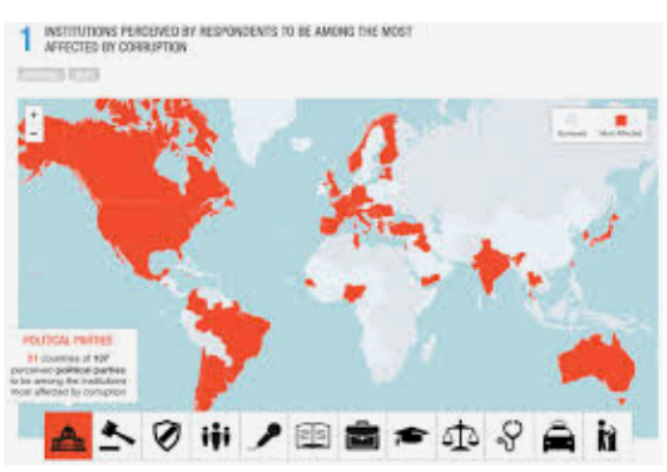

The Best Data Visualizations of All

datapine.com

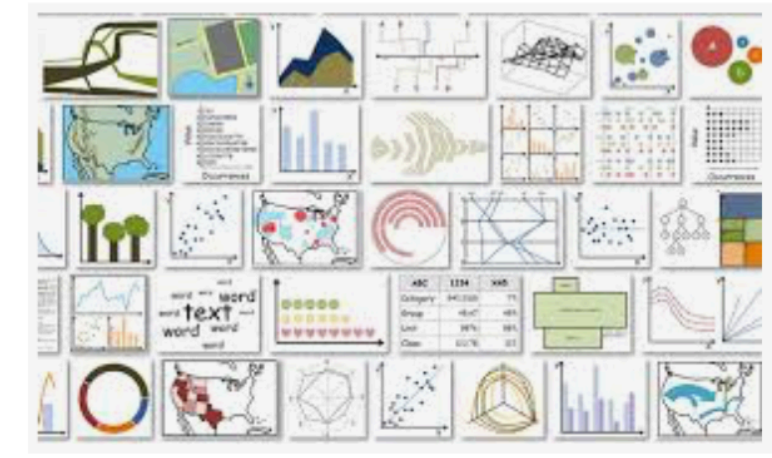

data visualization memorable

seas.harvard.edu

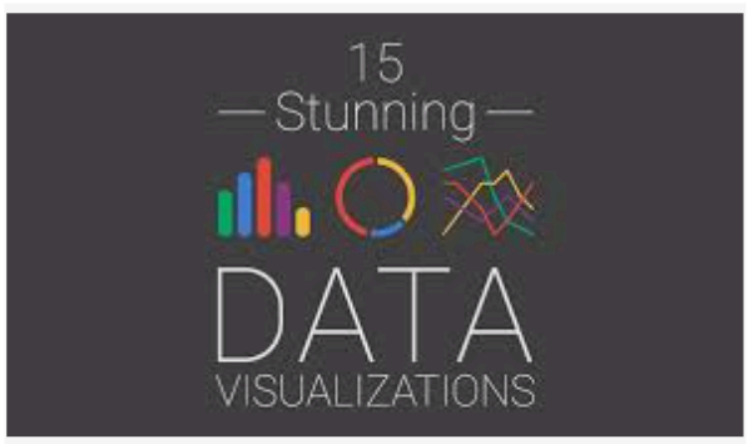

15 Stunning Data Visualizations (And blog.visme.co

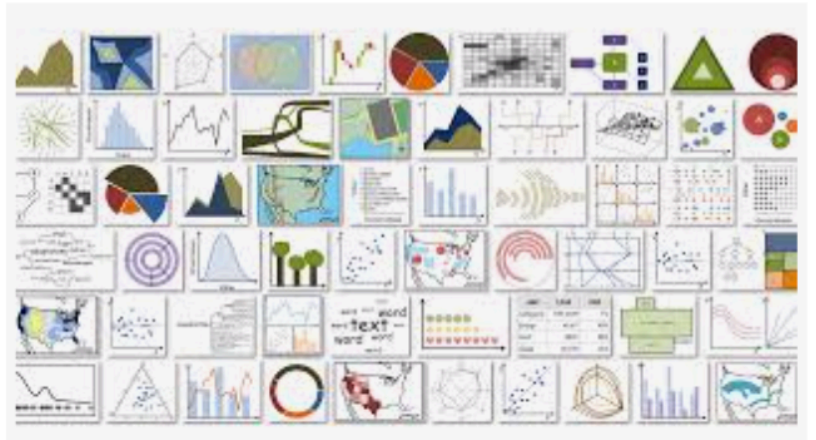

What makes a data visualization memorable? phys.org

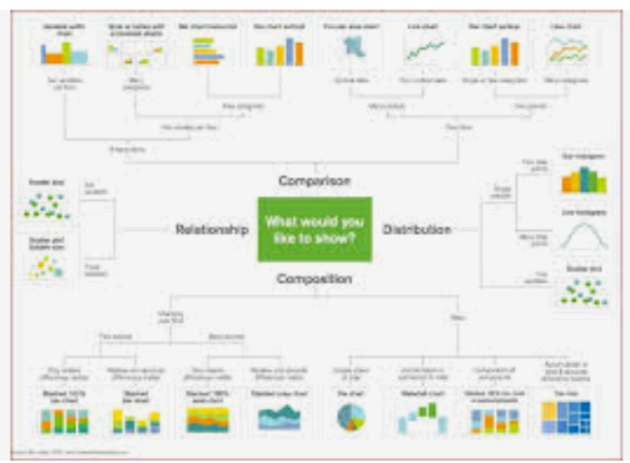

Data Visualizations in Python with Code towardsdatascience.com

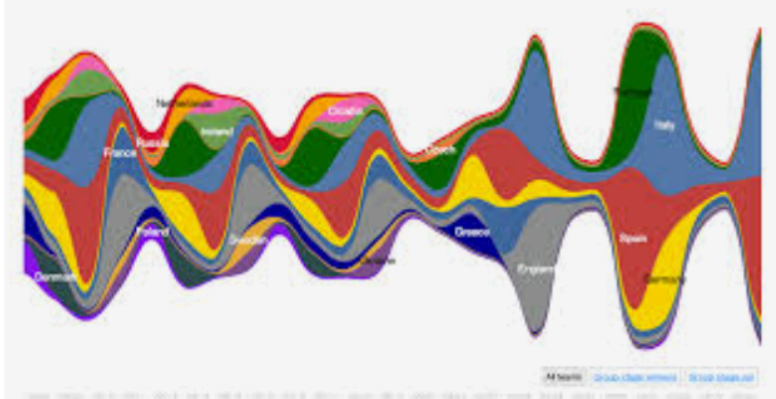

data density visualizations

radar.oreilly.com 


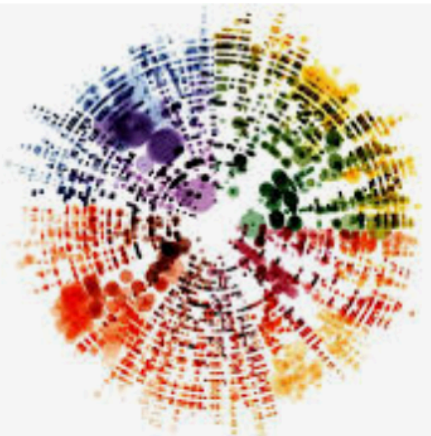

Tips for Data Visualization - Towards towardsdatascience.com

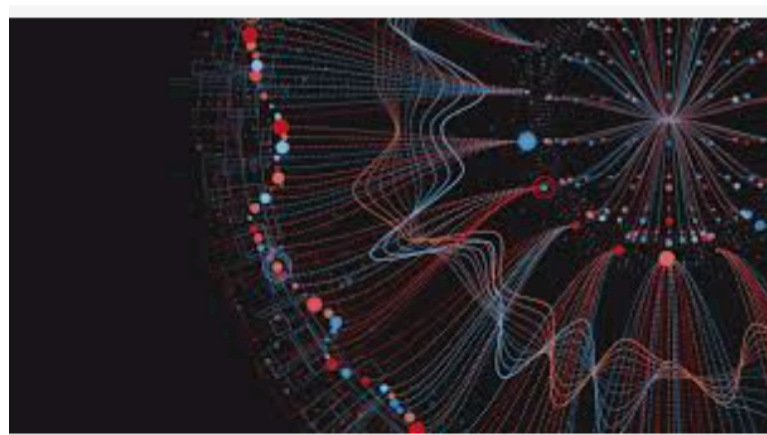

The importance of Data Visualisation nice.one

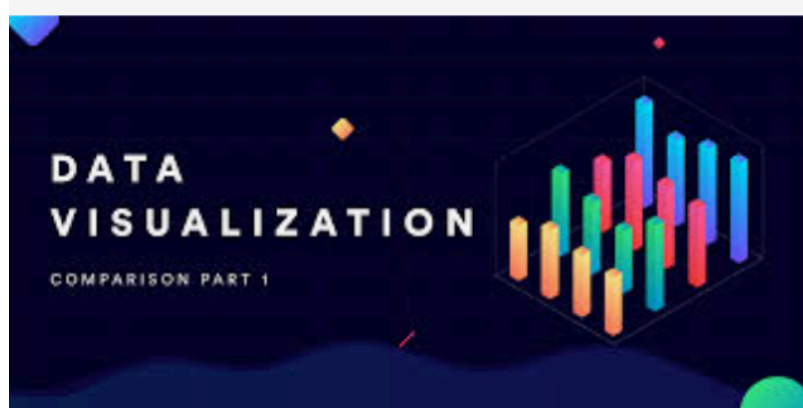

Your guide to Data Visualization for uxdesign.cc

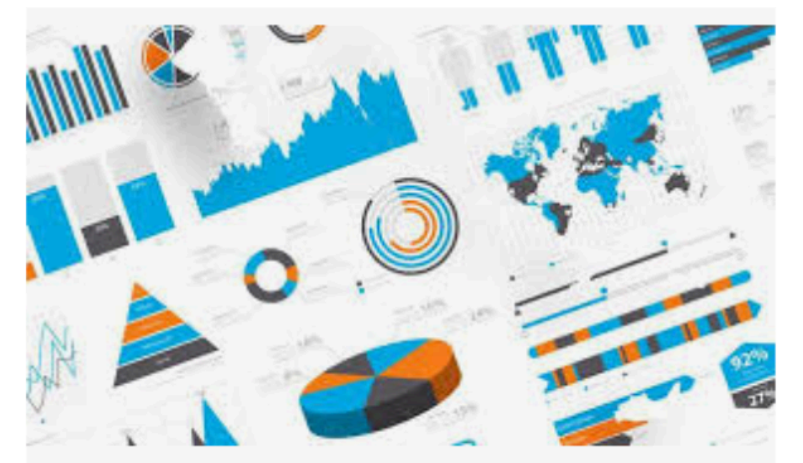

Choose the Right Data Visualization pcmag.com

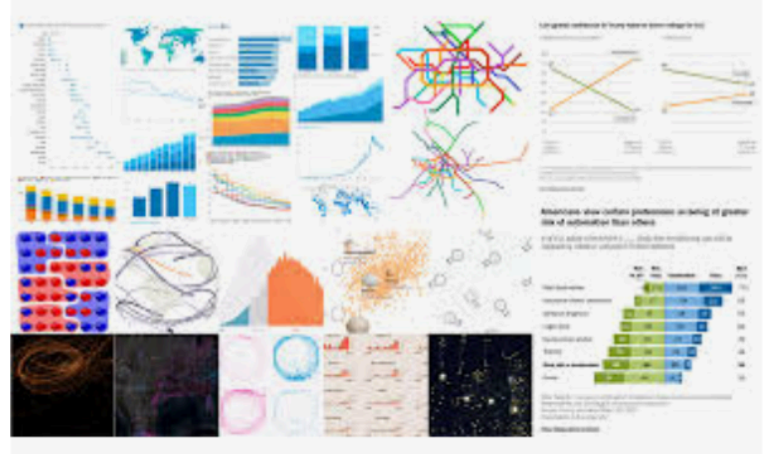

AnyChart | Data Visualization in 2017 anychart.com

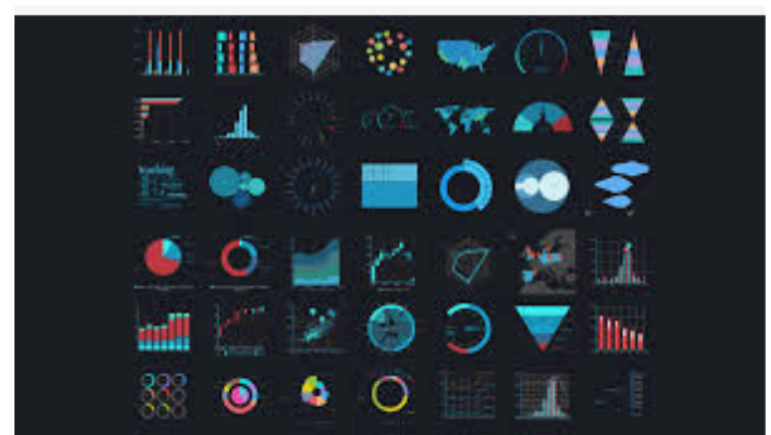

Coding for Data Visualization - Coding ... codinginthewild.com

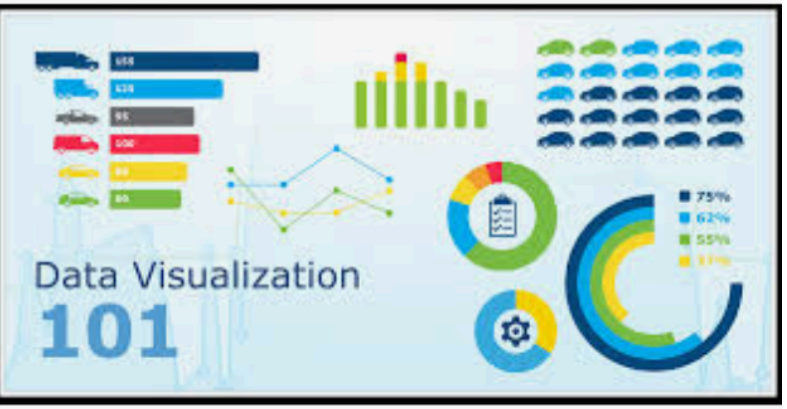

What is Data Visualization? | Geotab geotab.com

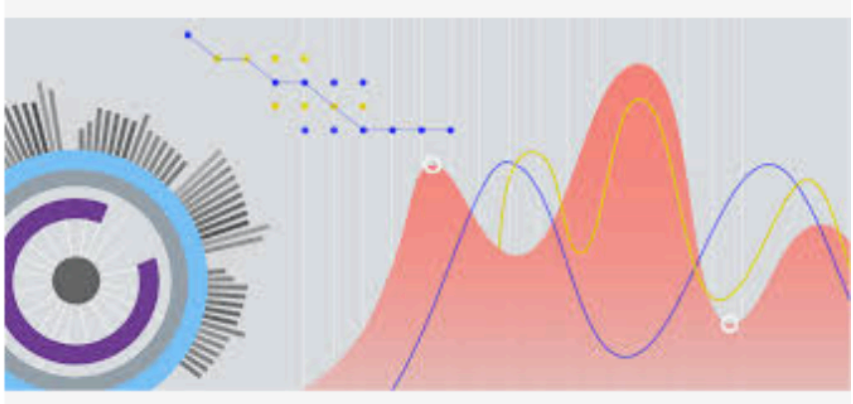

Data Visualization - Design at IBM medium.com
10 Big Data Visualization Tools promptcloud.com

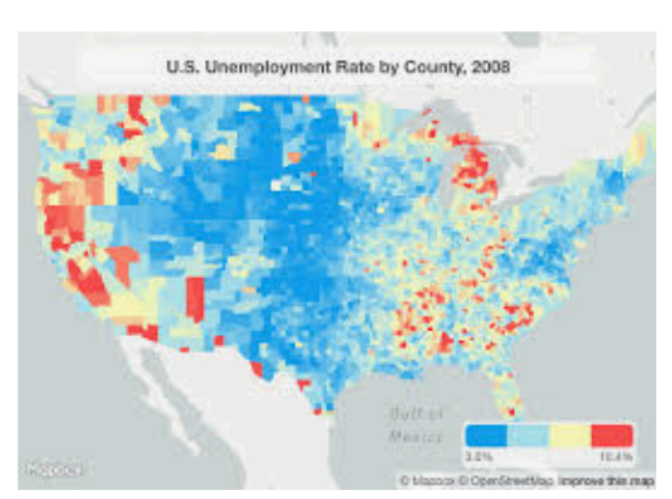

7 data visualization techniques for ... blog.mapbox.com

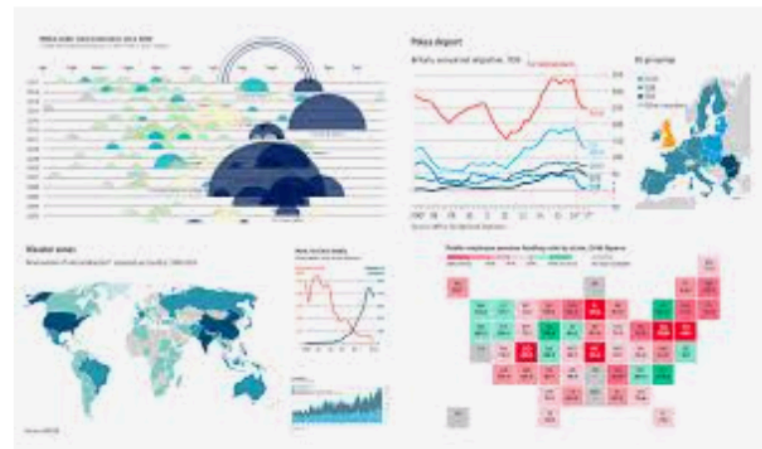

AnyChart | Visualizing Data in Charts anychart.com

Google Search for "Data Visualizations" 
2.

\title{
The Three Translations of Data Visualization
}

\author{
From World to Interpretation
}




\section{a) Datafication}

From World to Data 


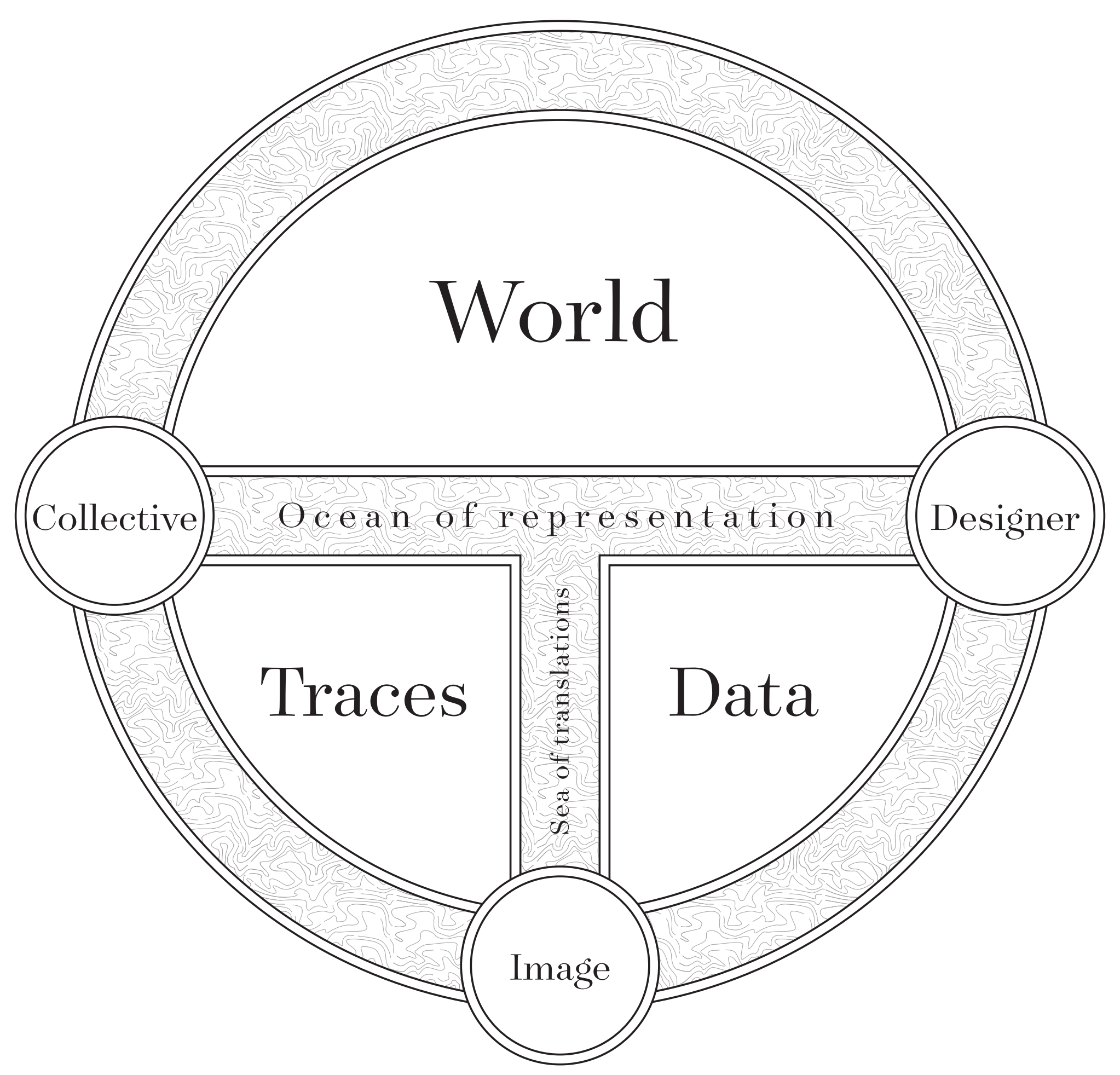




\title{
b) Visualization
}

\author{
From Data to Visualization
}




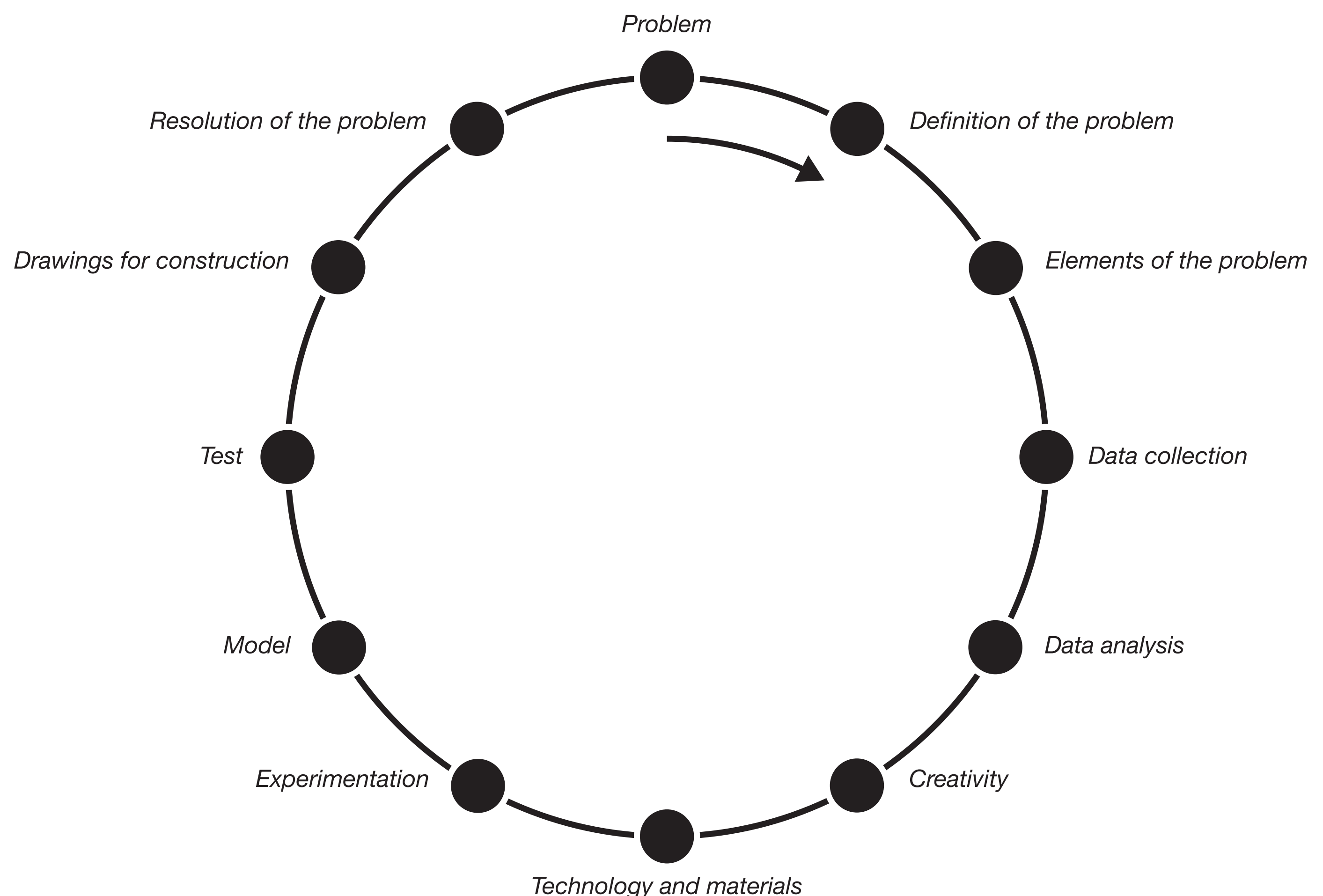

Design Process 


\title{
Elements
}

\author{
Points \\ Lines \\ Shapes \\ Volumes \\ Texts
}




\title{
Element Characteristics
}

\author{
Size \\ Position \\ Color \\ Transparency
}

Font 


\title{
Structures
}

\author{
Hierarchical (trees) \\ Relational (networks) \\ Temporal (timelines)
}

Spatial (maps)

Textual (books) 


\section{c) Interpretation}

From Visualization to Interpretation 


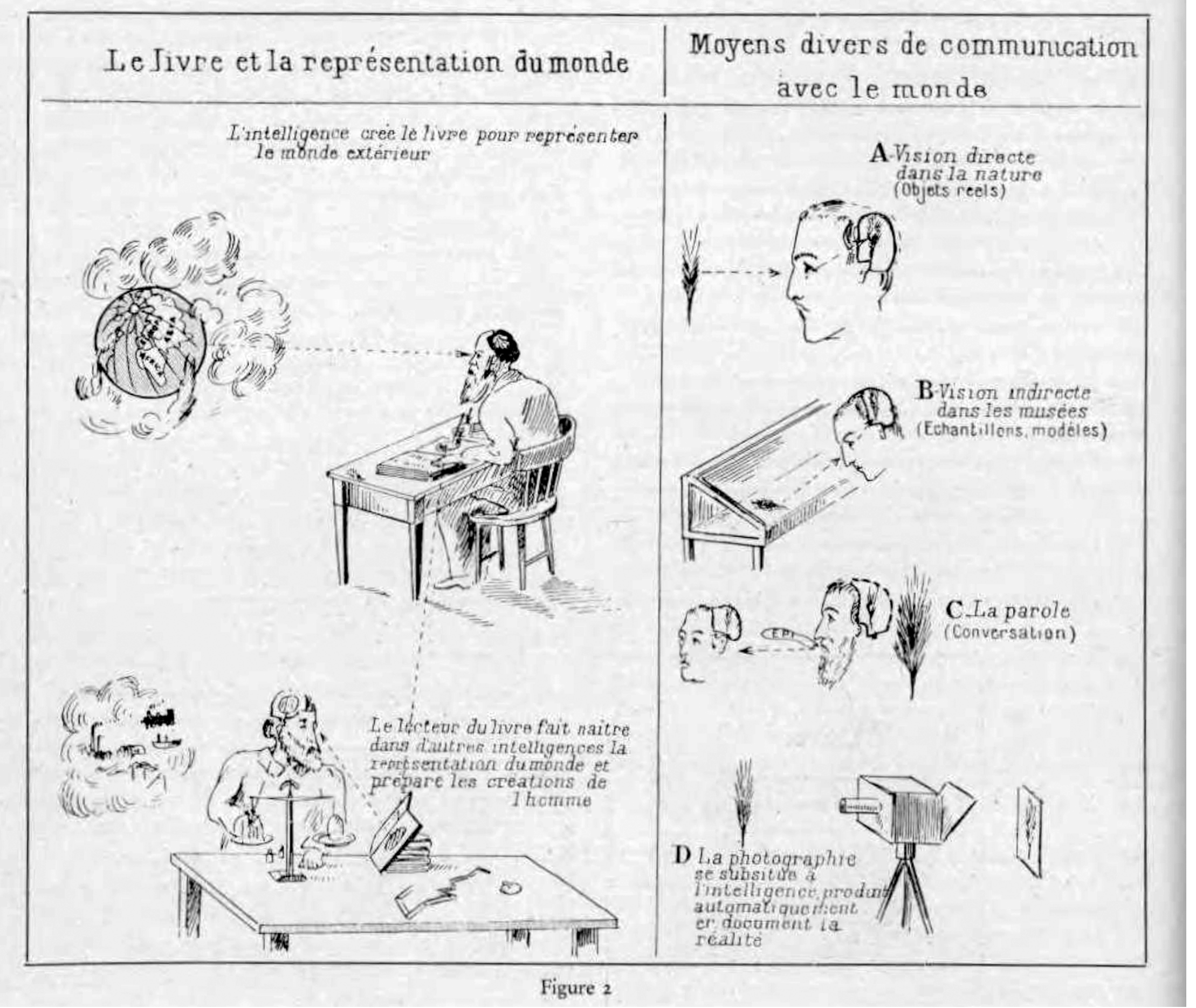

Traité de documentation : le livre sur le livre, théorie et pratique, Paul Otlet (1934) 


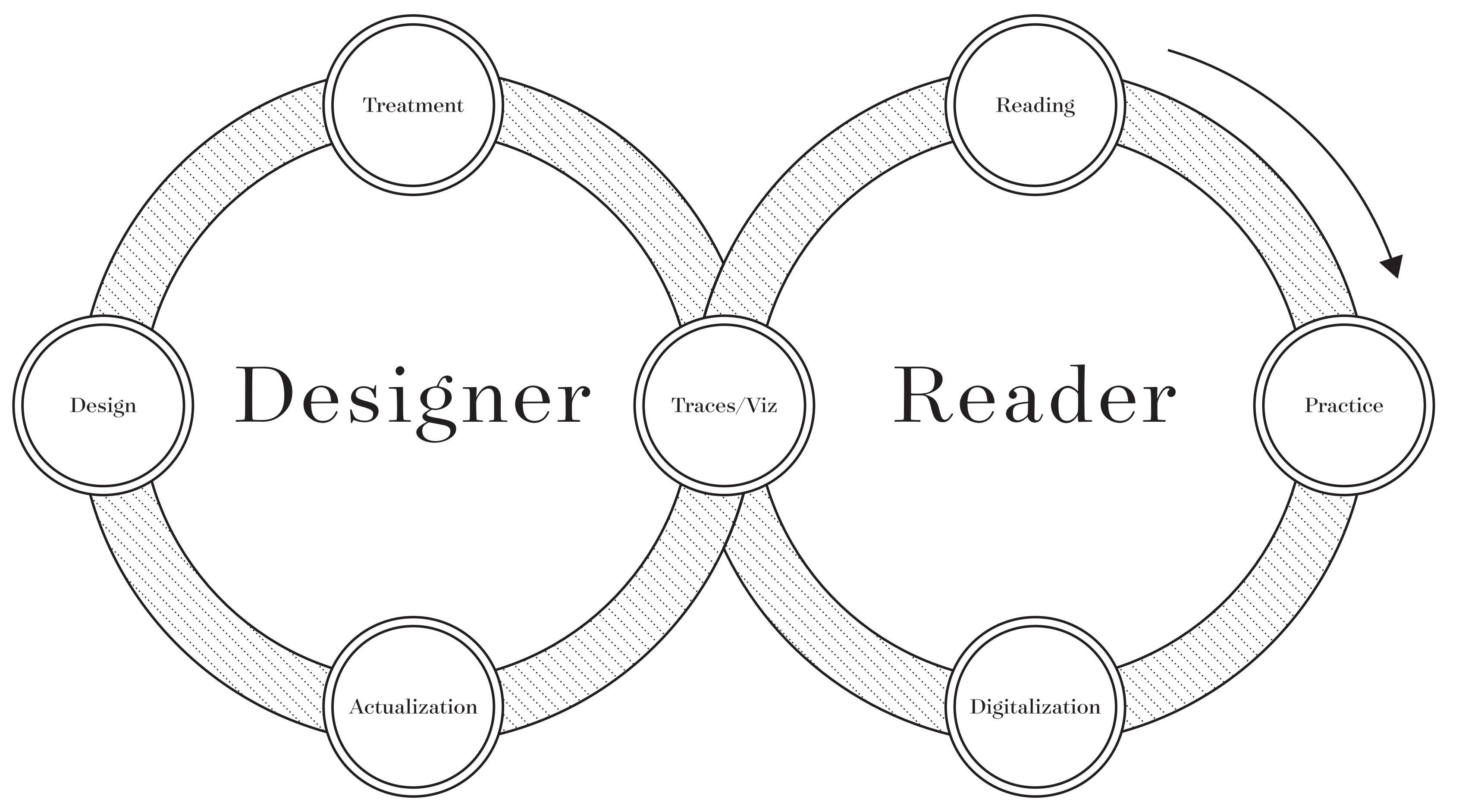

Rodighiero \& Romele (2018) 


\title{
Interpretation
}

\author{
Association
}

Differentiation 


\section{3.}

\section{Examples}

Classical Visualizations (18th-19th centuries)

Modern Visualizations (20th century)

Contemporary Visualizations (21st century) 


\section{a.}

\section{Classical Visualizations}




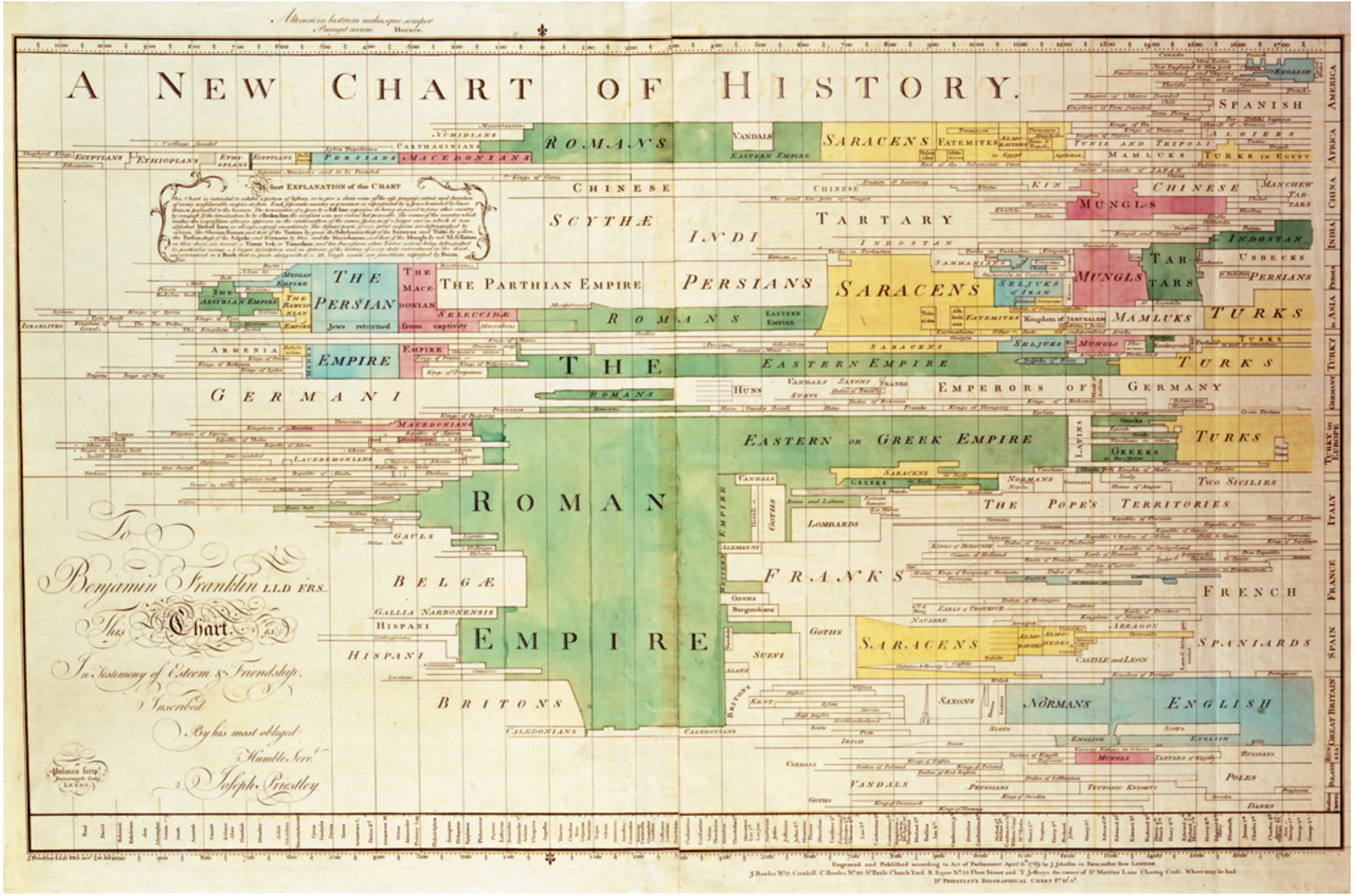

A New Chart of History, Joseph Priestley (1769) https://en.wikipedia.org/wiki/Joseph_Priestley 
Exports and Imports to and from DENMARK \&e NORWAY from 1700 to 1780.

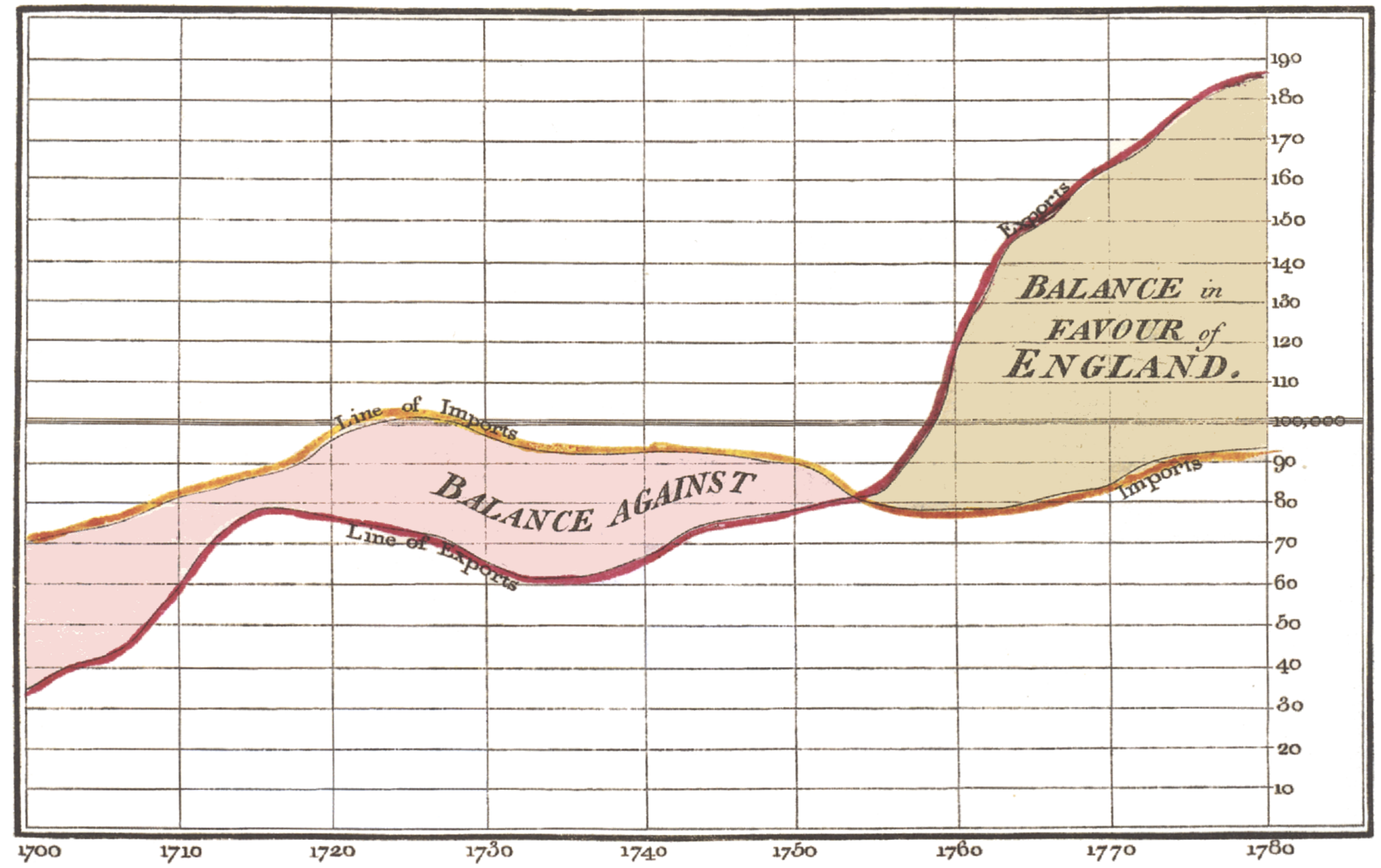

Commercial and Political Atlas, William Playfair (1786)

https://en.wikipedia.org/wiki/William_Playfair 


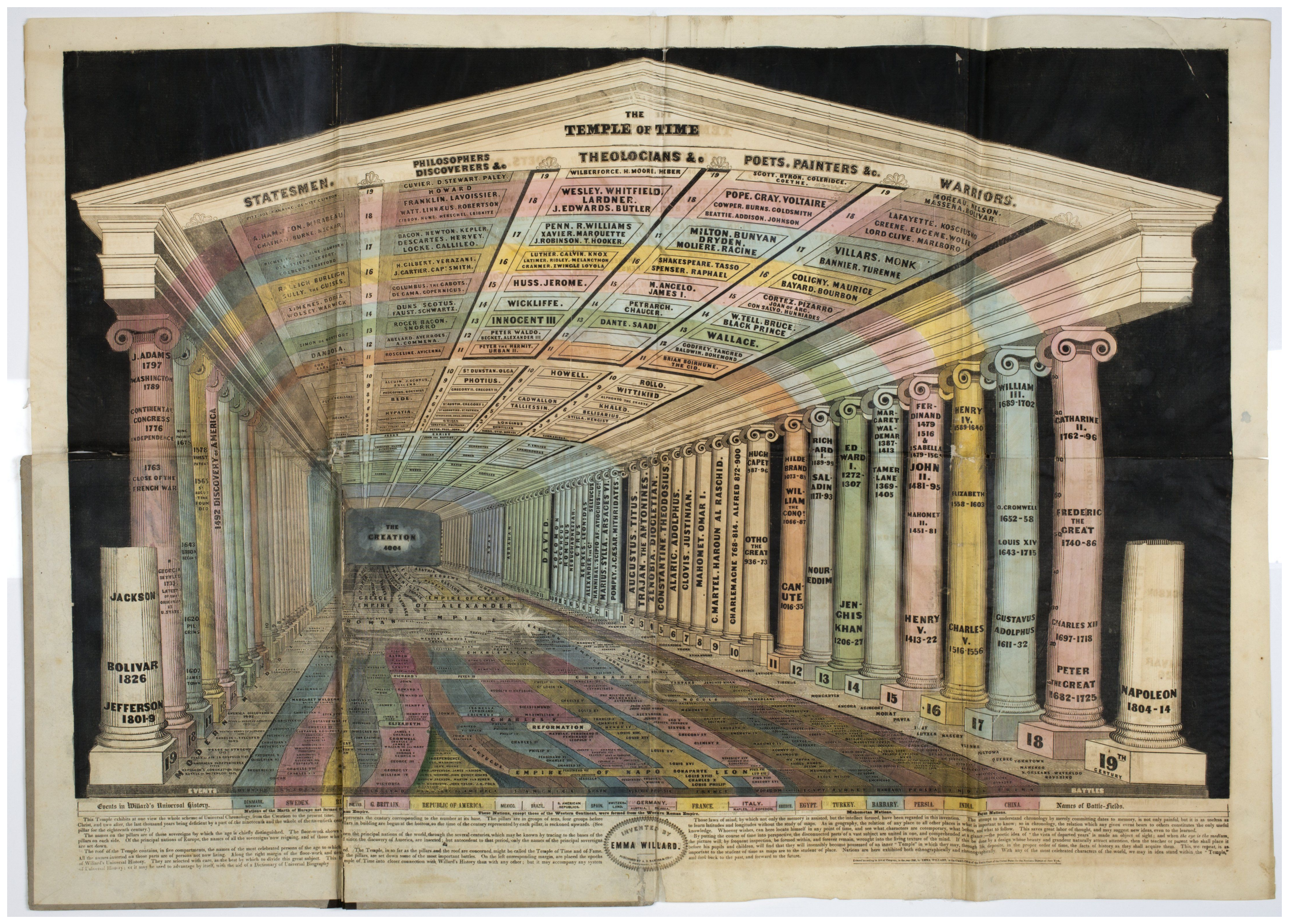

Temple of Time, Emma Willard (1846) https://en.wikipedia.org/wiki/Emma_Willard 


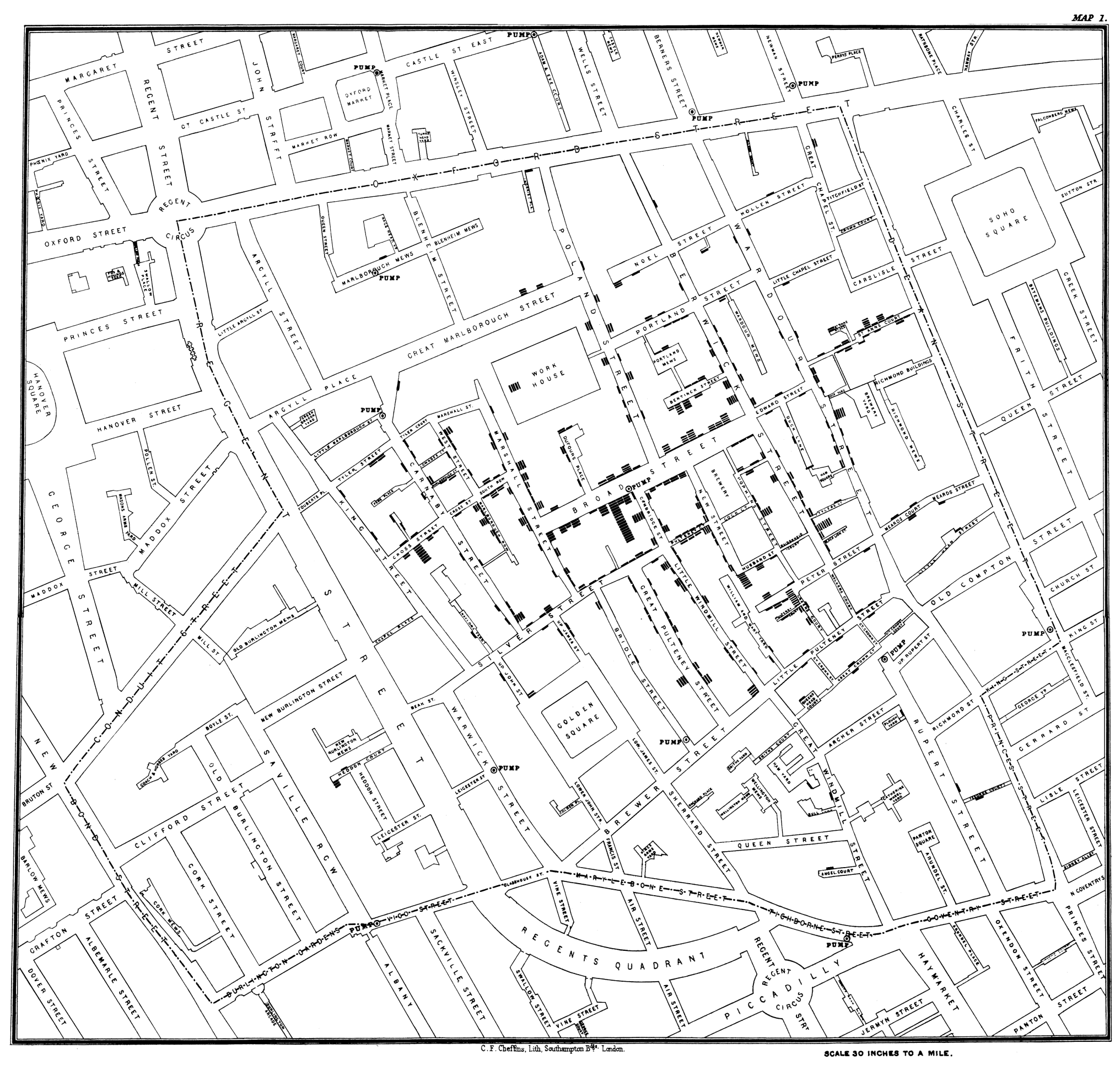

Clusters of cholera cases in the London, John Snow (1854) https://en.wikipedia.org/wiki/John_Snow 


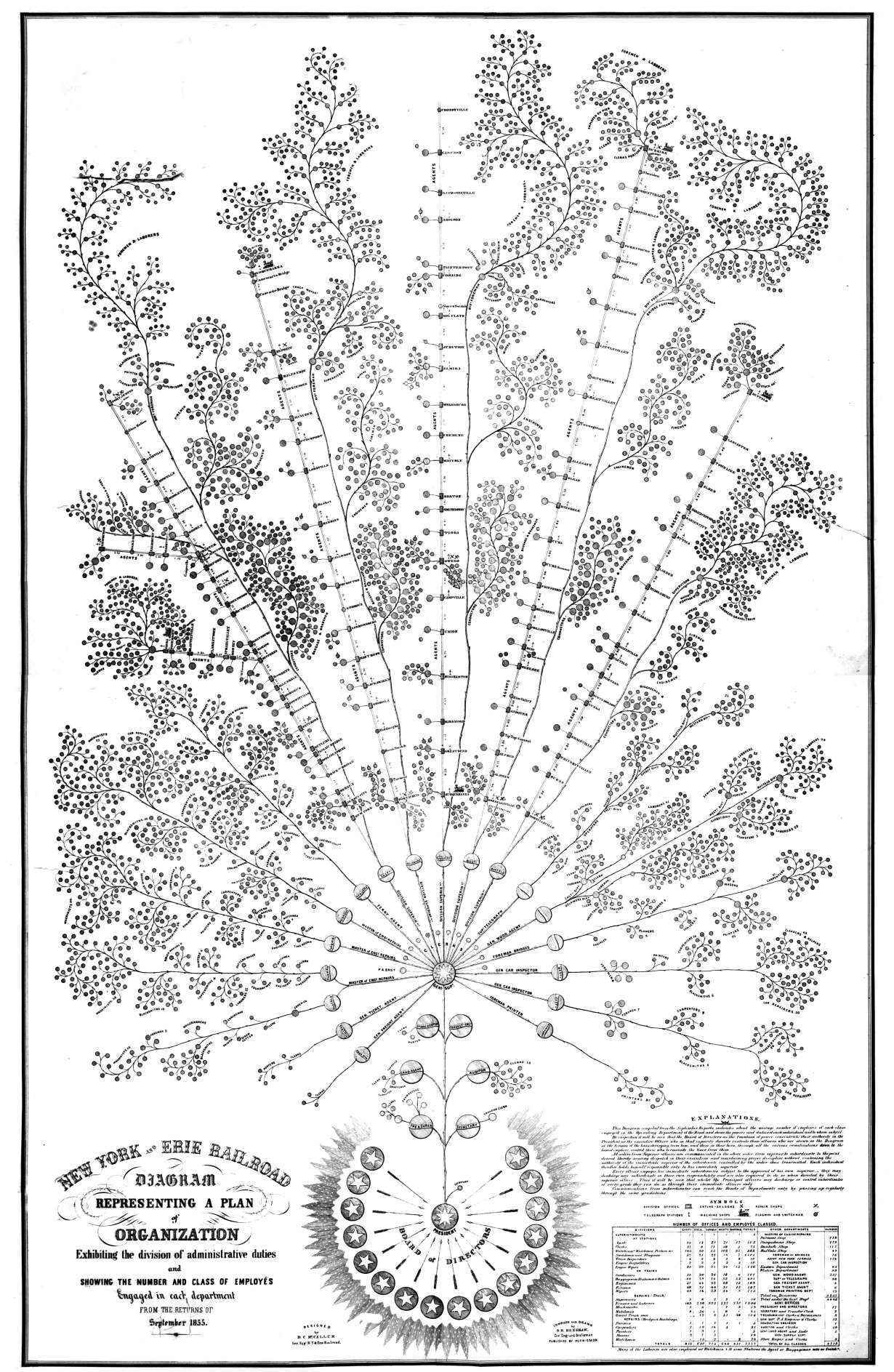

Organizational diagram of the New York and Erie Railroad, Daniel McCallum (1855) https://en.wikipedia.org/wiki/Daniel_McCallum 
APRTL 1855 T0 MARCH 1856

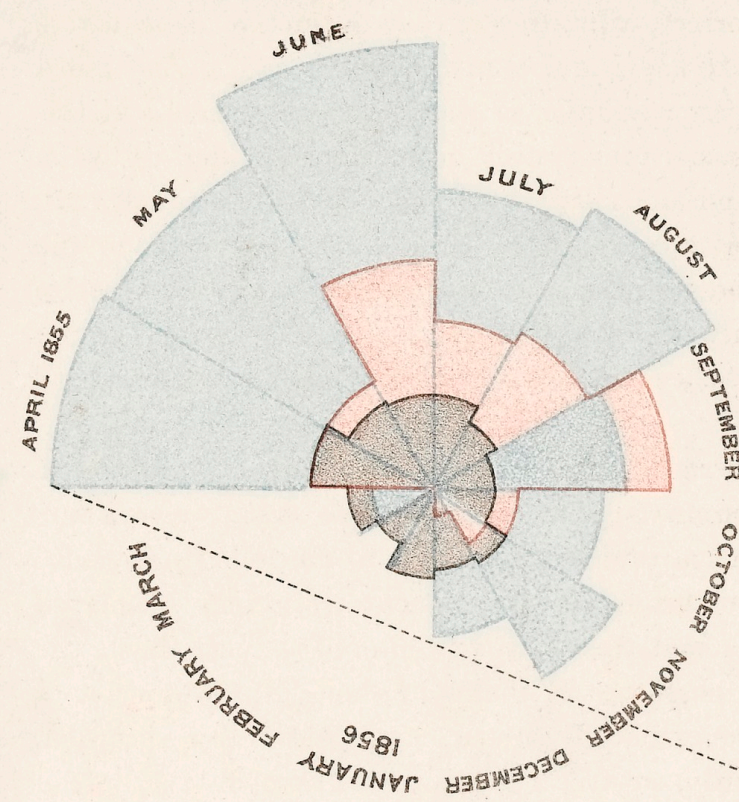

The Areas of the blue red, \& black wedges are each measured from the centre as the common nertex.

The blue inedges. measured from the centre of the circle represent area for area the deaths from Preacntible or Mitigable. Zymotic diseases, the red voedges measured from the centre the deaths from soounds. \& the black toedges measured from the centre the deaths from all other cause. The black tine across the red triangle in Non" 1854 marts the boundary of the deaths from all other causes during the month

In October 1854, \& Aprut 1855, the black area rooinesides with the red, in January \& February 1855, the blue coincides with the blach

The entire areas may be compared by following the blue, the red \& the brack tines enclosing them.
APRIL 1854 T0 MARCH 1855. 


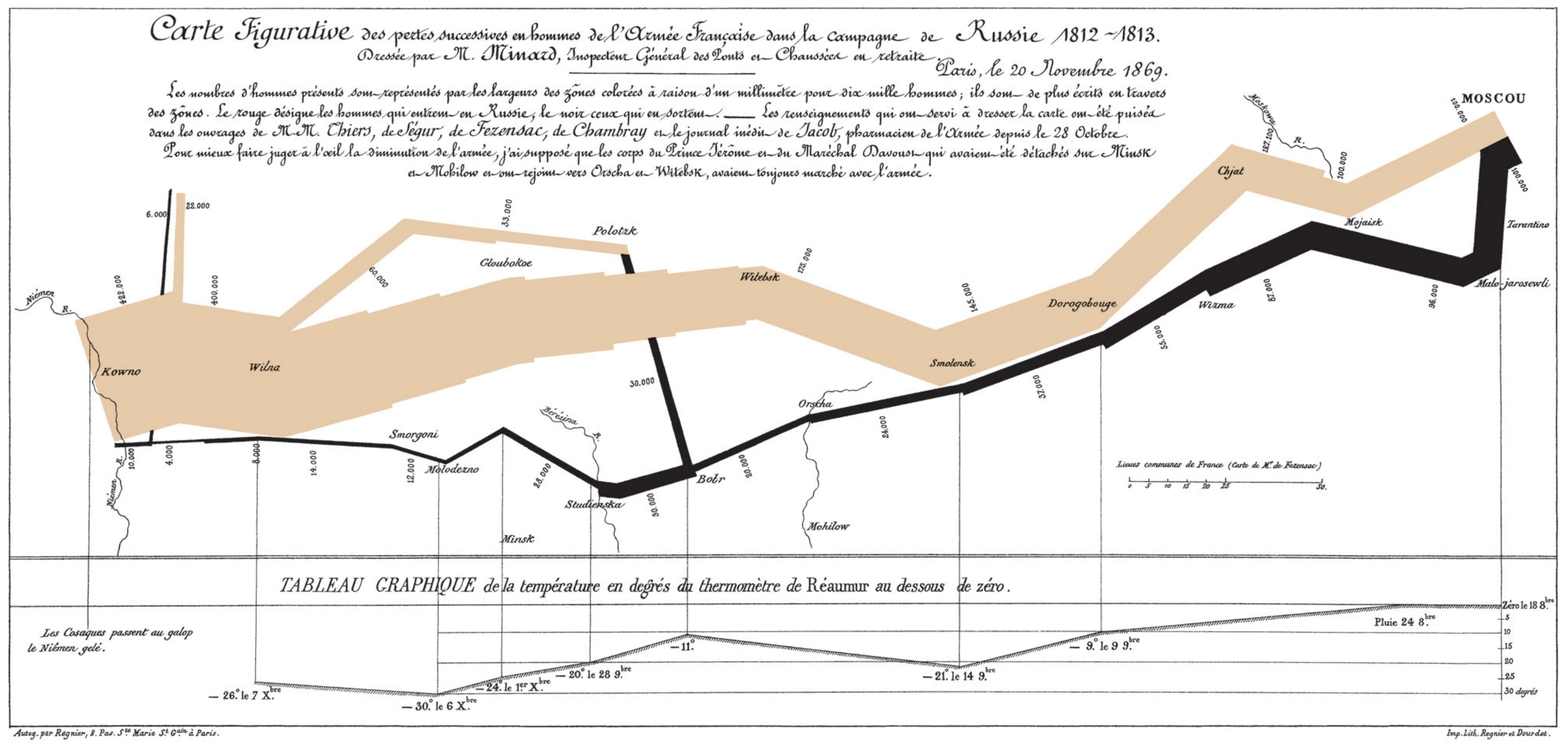

Campagne de Russie, Charles Joseph Minard (1861) https://en.wikipedia.org/wiki/Charles_Joseph_Minard 


\section{b.}

\section{Modern Visualizations}




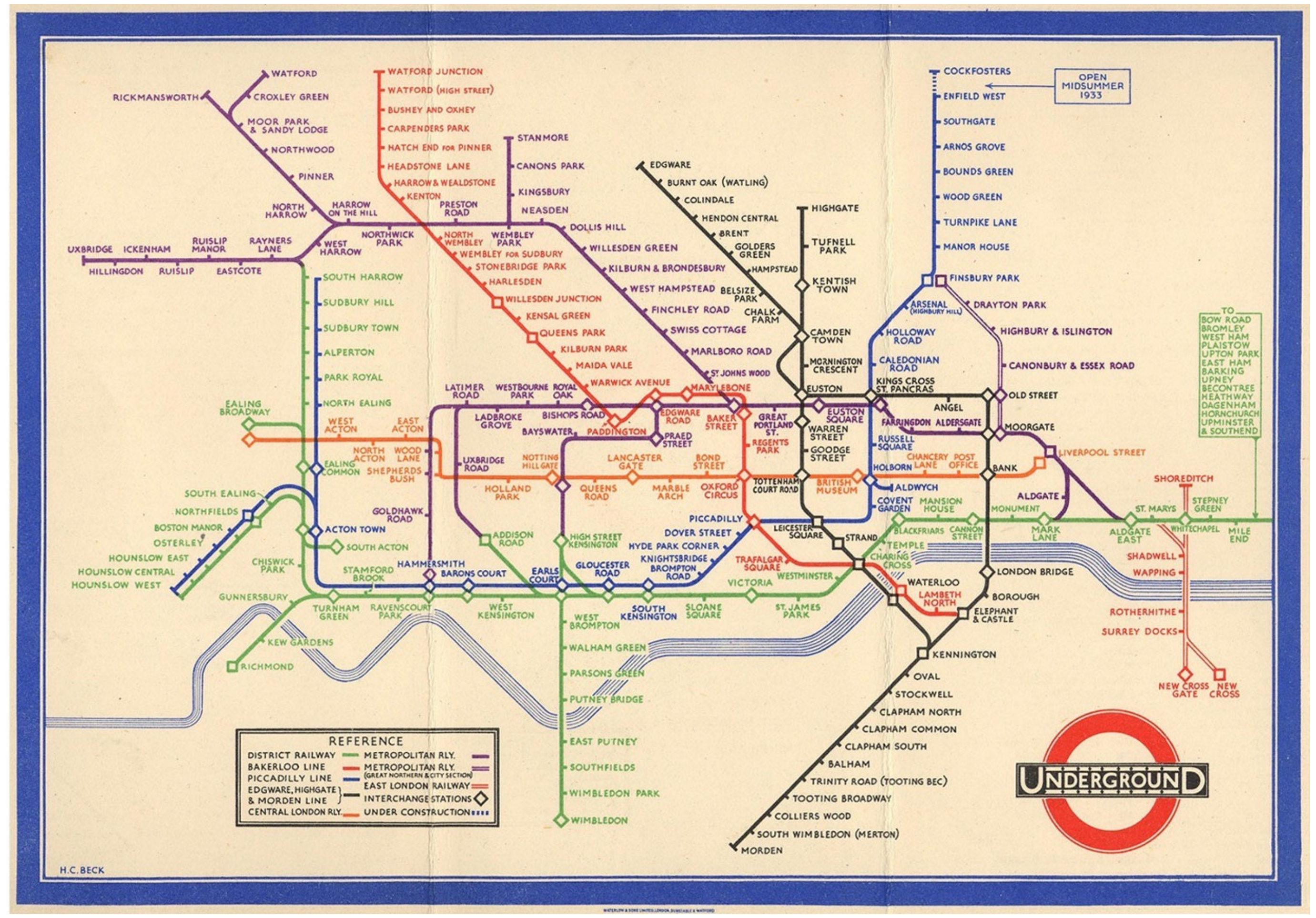

London Underground Map, Harry Beck (1933) https://en.wikipedia.org/wiki/Harry_Beck 


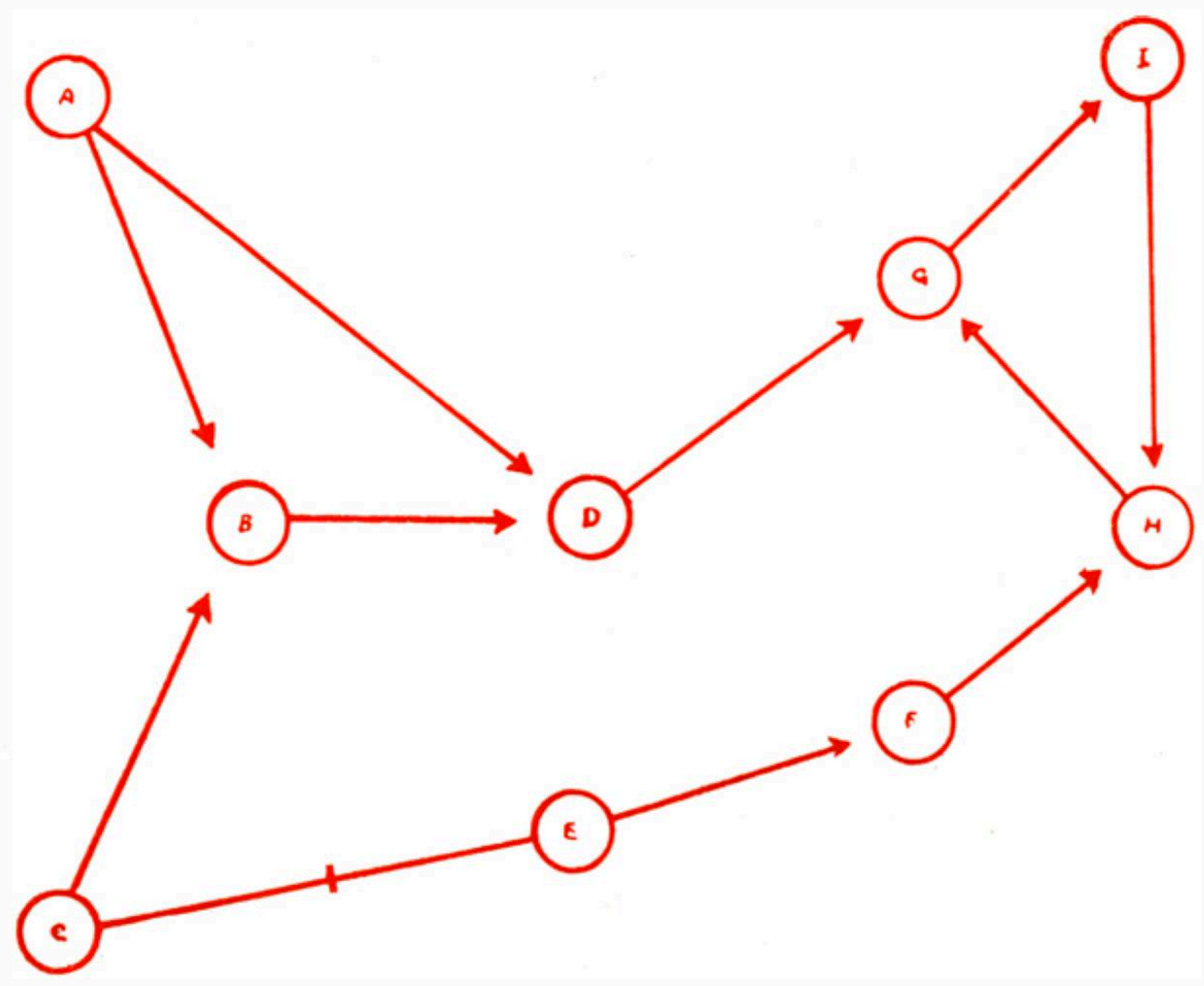

Sociograms, Jacob L. Moreno (1934)

https://en.wikipedia.org/wiki/Jacob_L. Moreno 


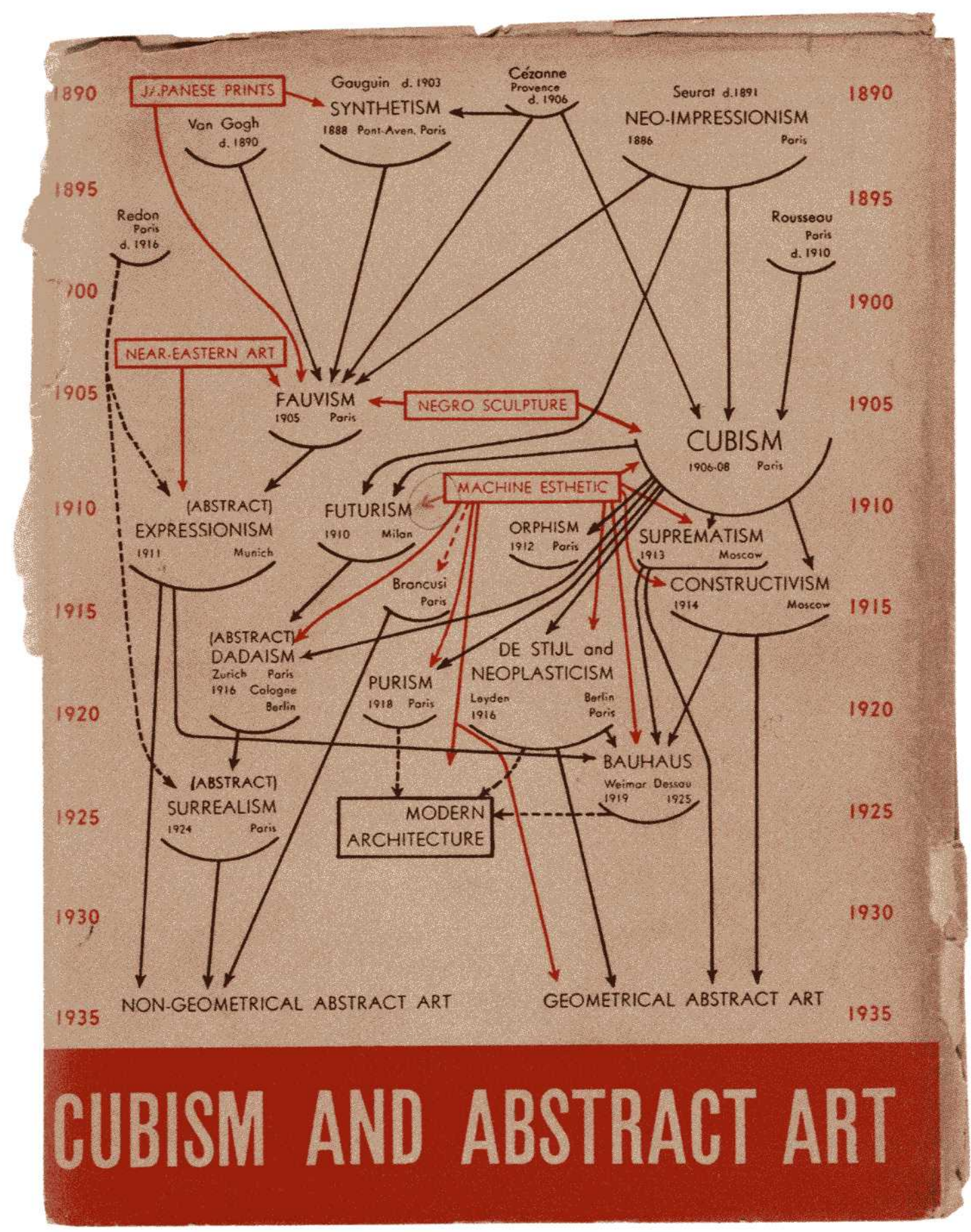

Cubism and Abstract Art, Alfred Barr (1936) https://www.moma.org/calendar/exhibitions/2748 
1820

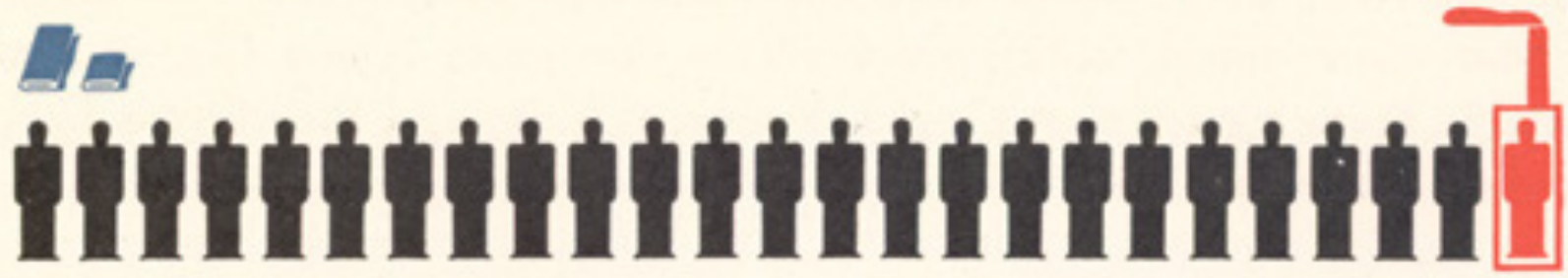

1830

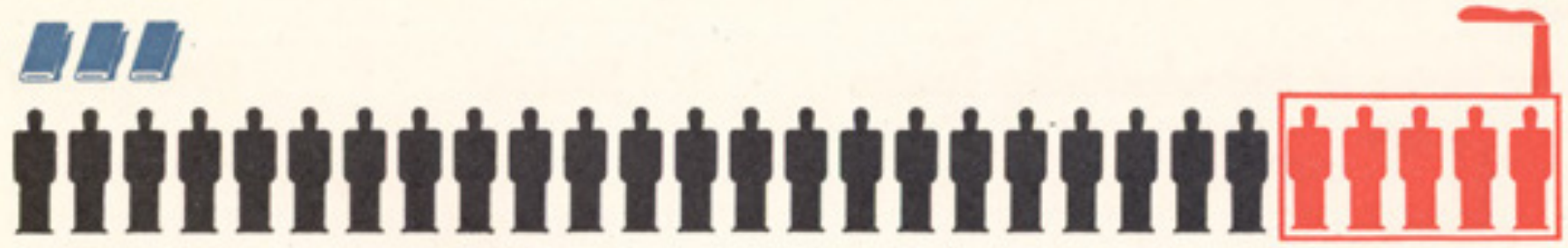

1845

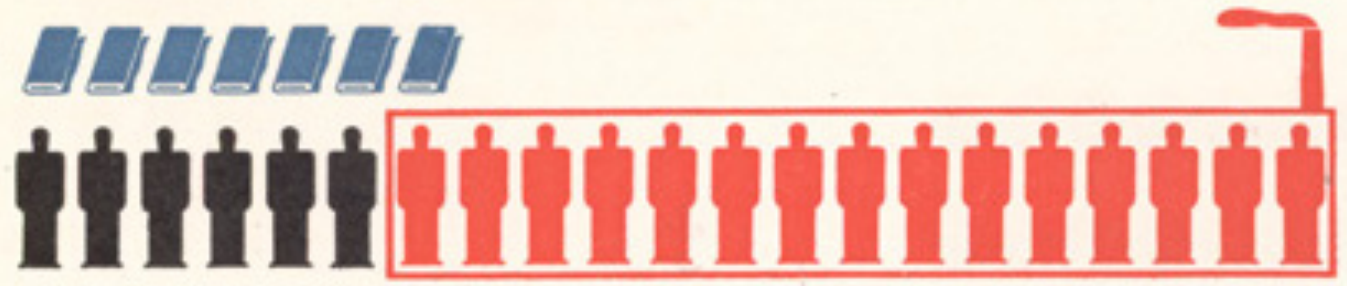

1860

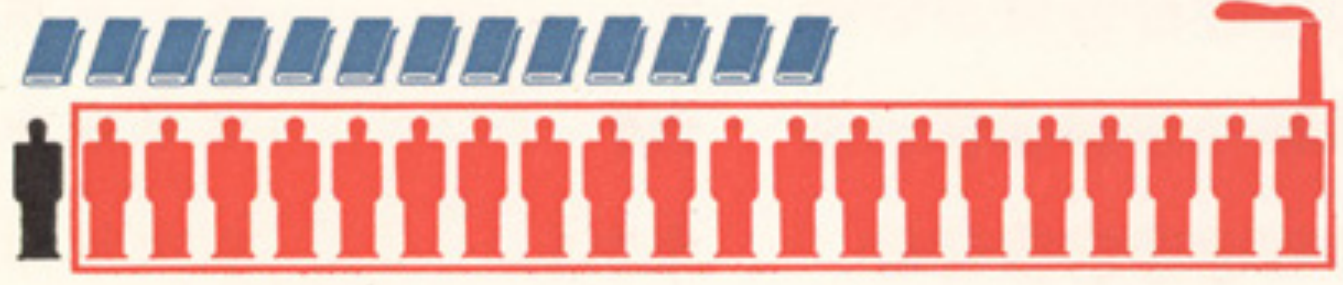

1880

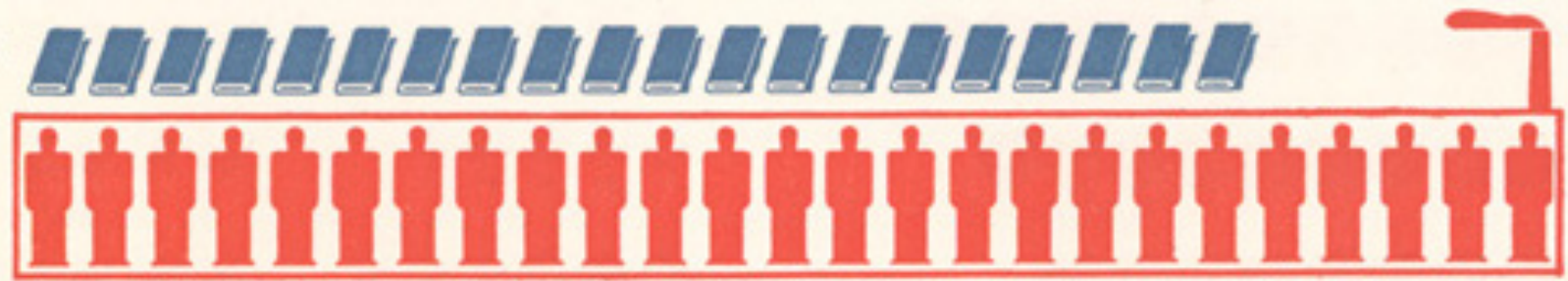

Each blue symbol represents $\mathbf{5 0}$ million pounds total production

Each black man symbol represents 10,000 home weavers

Each red man symbol represents 10,000 factory weavers

Modern Man in the Making - Isotype, Otto Neurath (1939)

https://en.wikipedia.org/wiki/Otto Neurath 


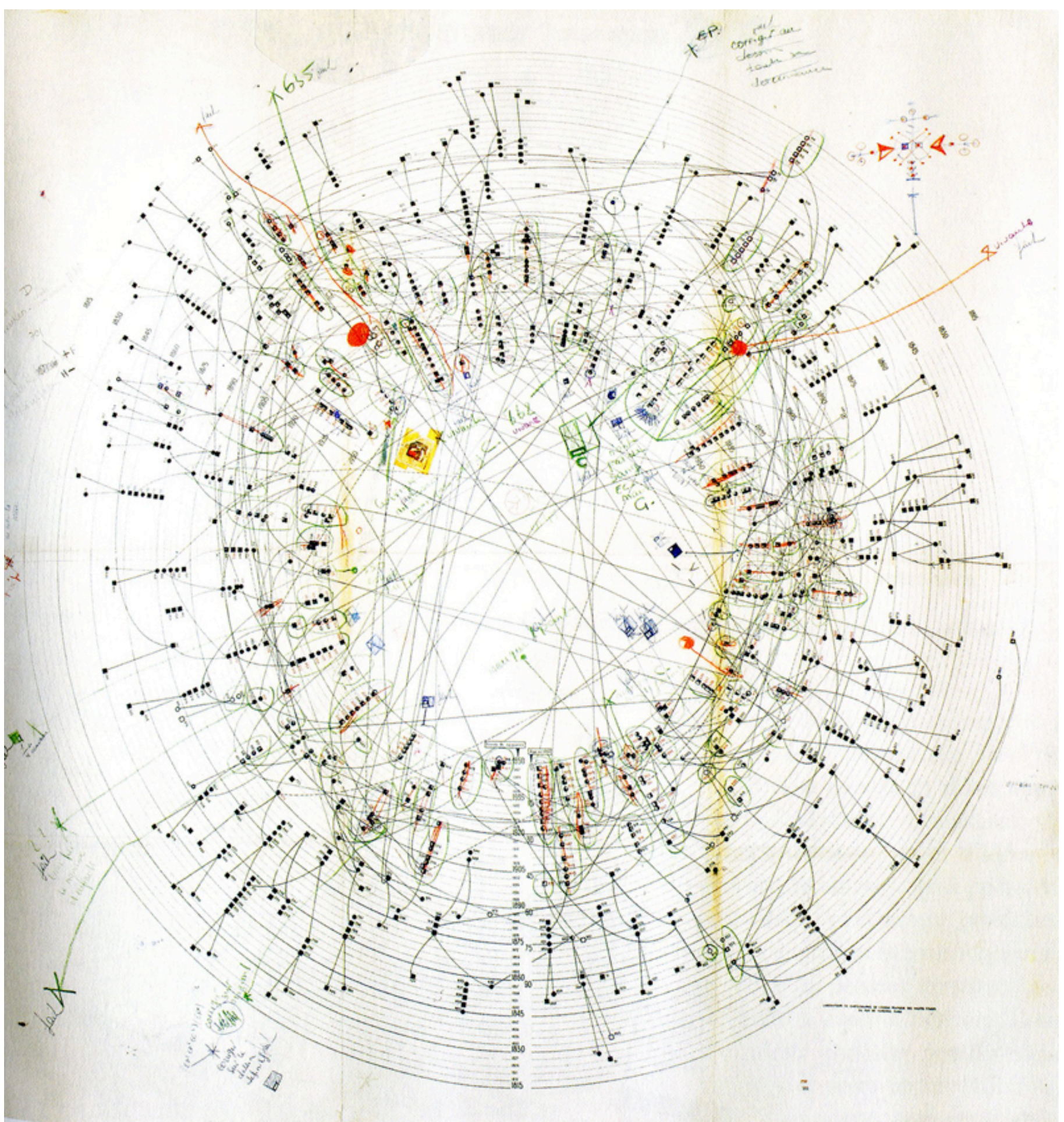

Genealogical diagram, Jean Malaurie (1950s) https://en.wikipedia.org/wiki/Jean_Malaurie 


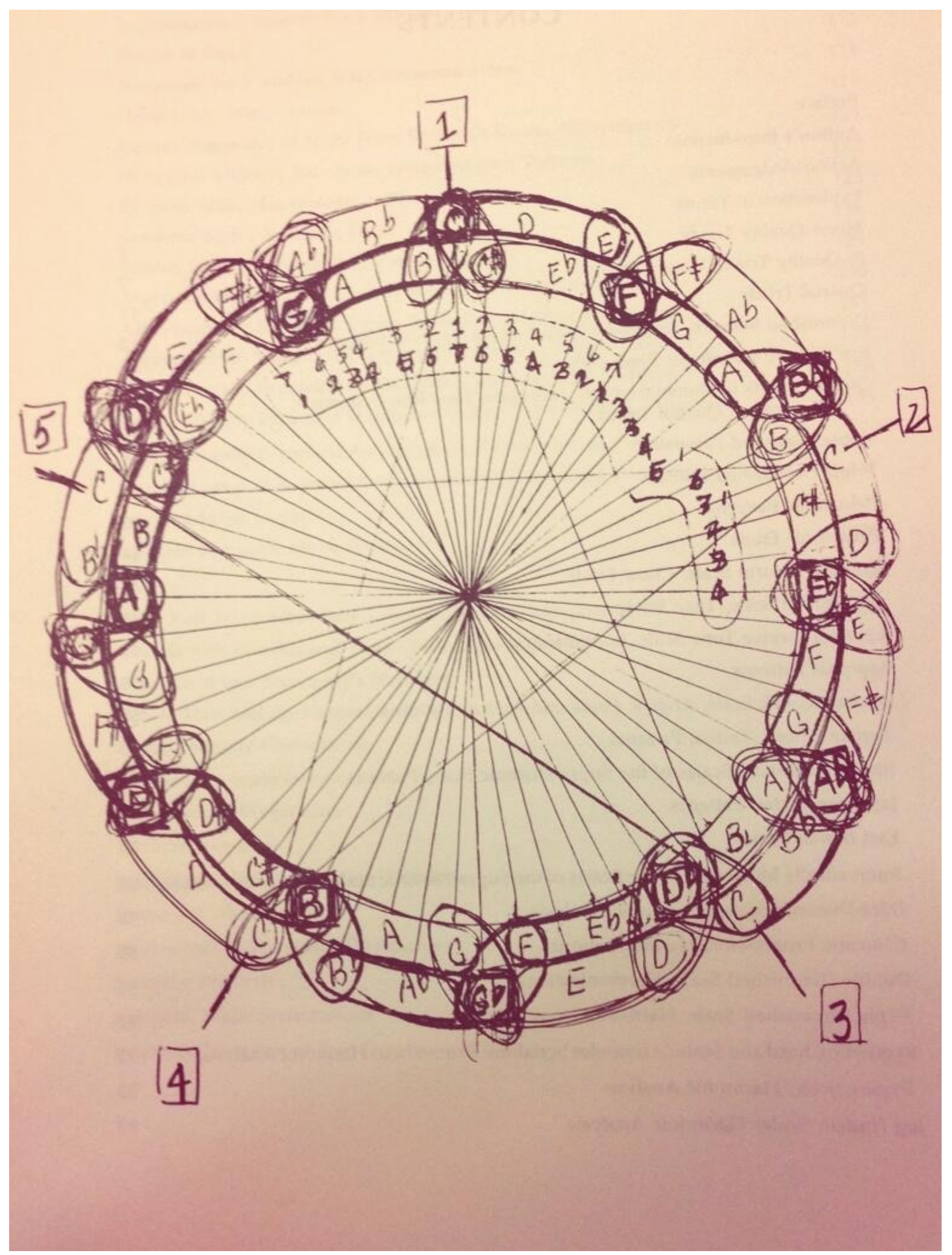

Circle of Fifths, John Coltrane (1967)

http://www.openculture.com/2017/04/the-tone-circle-john-coltrane-drewto-illustrate-the-theory-behind-his-most-famous-compositions-1967.html 


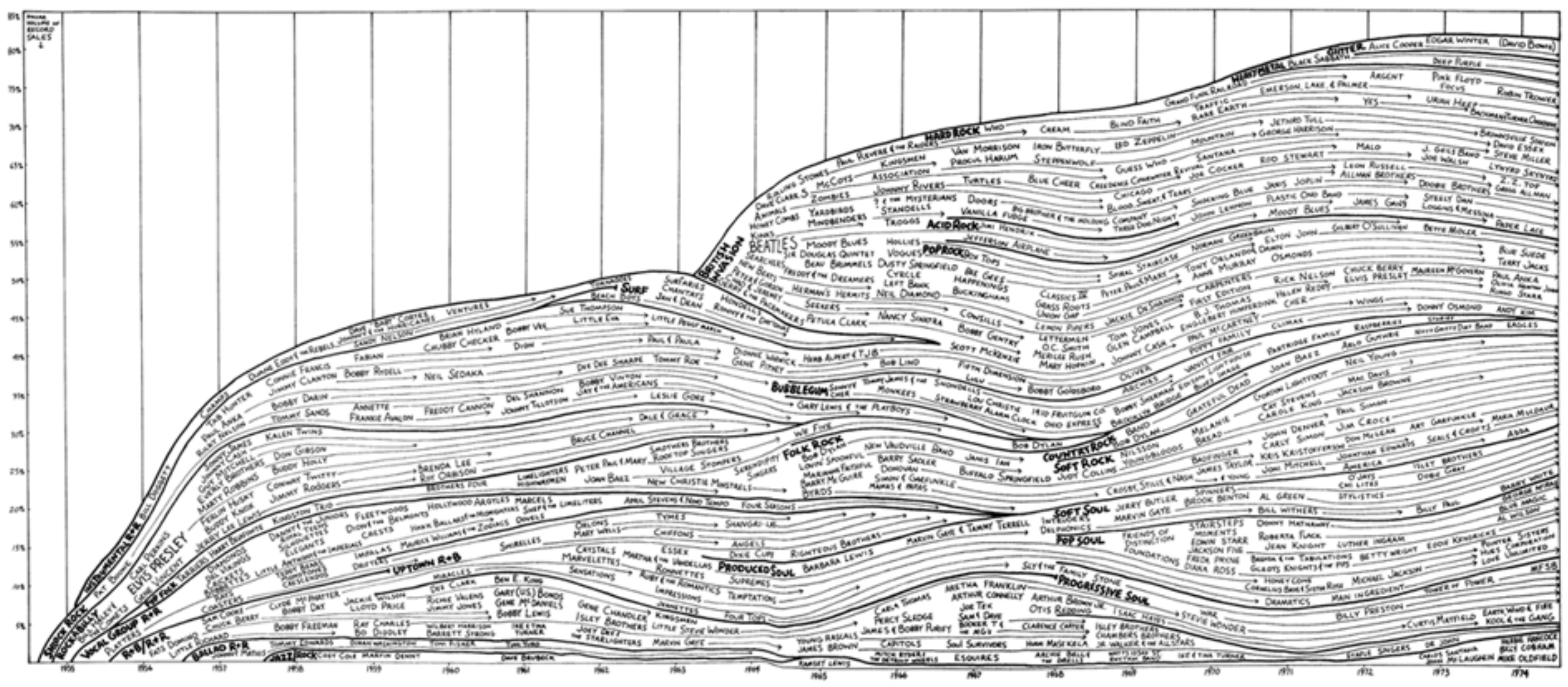

The History of Rock, Reebee Garofalo (1975)

https://www.edwardtufte.com/bboard/q-and-a-fetch-msg?msg_id=0002N4 


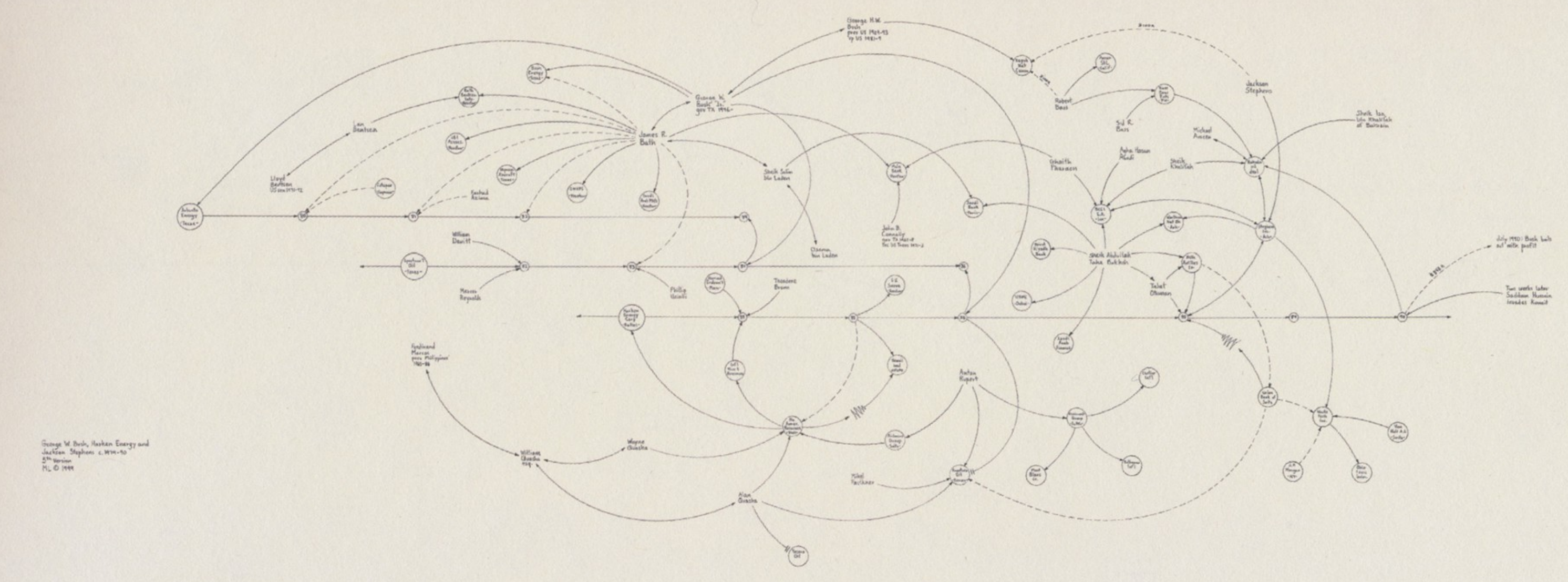

George W. Bush, Harken Energy, and Jackson Stephens, Mark Lombardi (1979-90) https://medium.com/@ben_fry/learning-from-lombardi-a28032a7eb5 


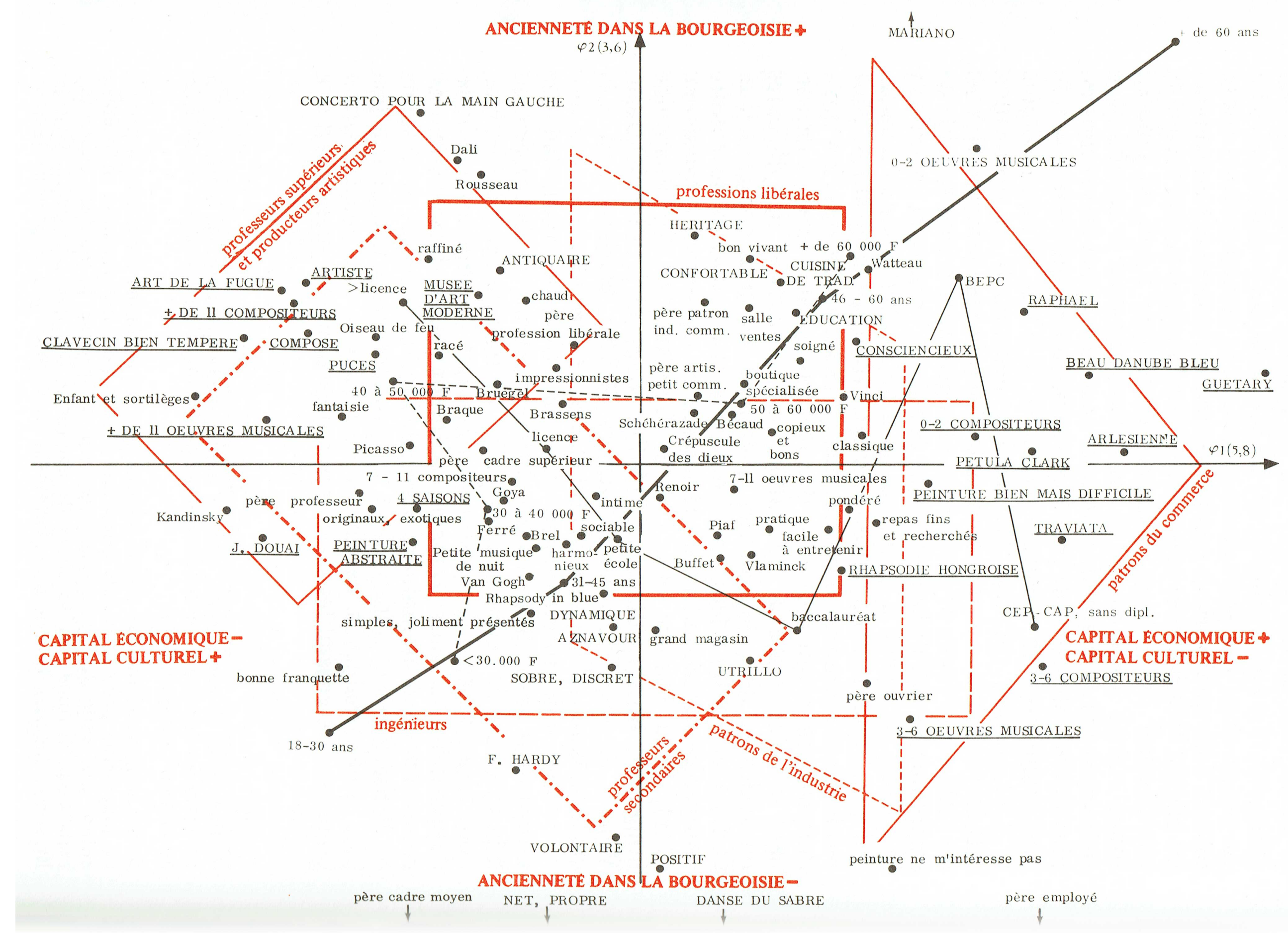

Multiple Correspondence Analysis (MCA) in La distinction, Pierre Bourdieu (1979) https://en.wikipedia.org/wiki/Multiple correspondence analysis 


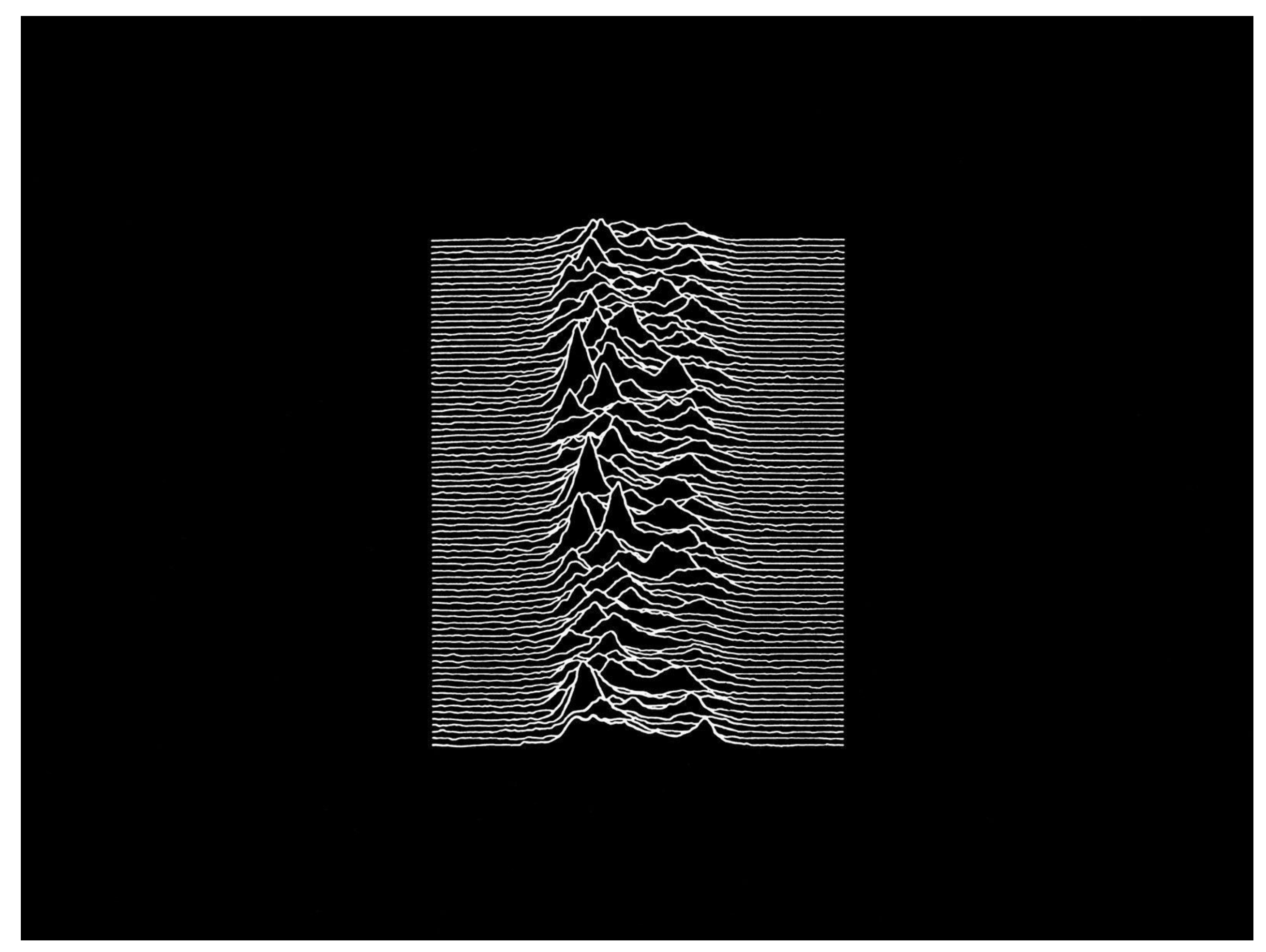

Stacked Plot of Radio Signals from a Pulsar, Harold Craft (1977) https://blogs.scientificamerican.com/sa-visual/pop-culture-pulsar-originstory-of-joy-division-s-unknown-pleasures-album-cover-video/ 


\section{c.}

\section{Contemporary Visualizations}




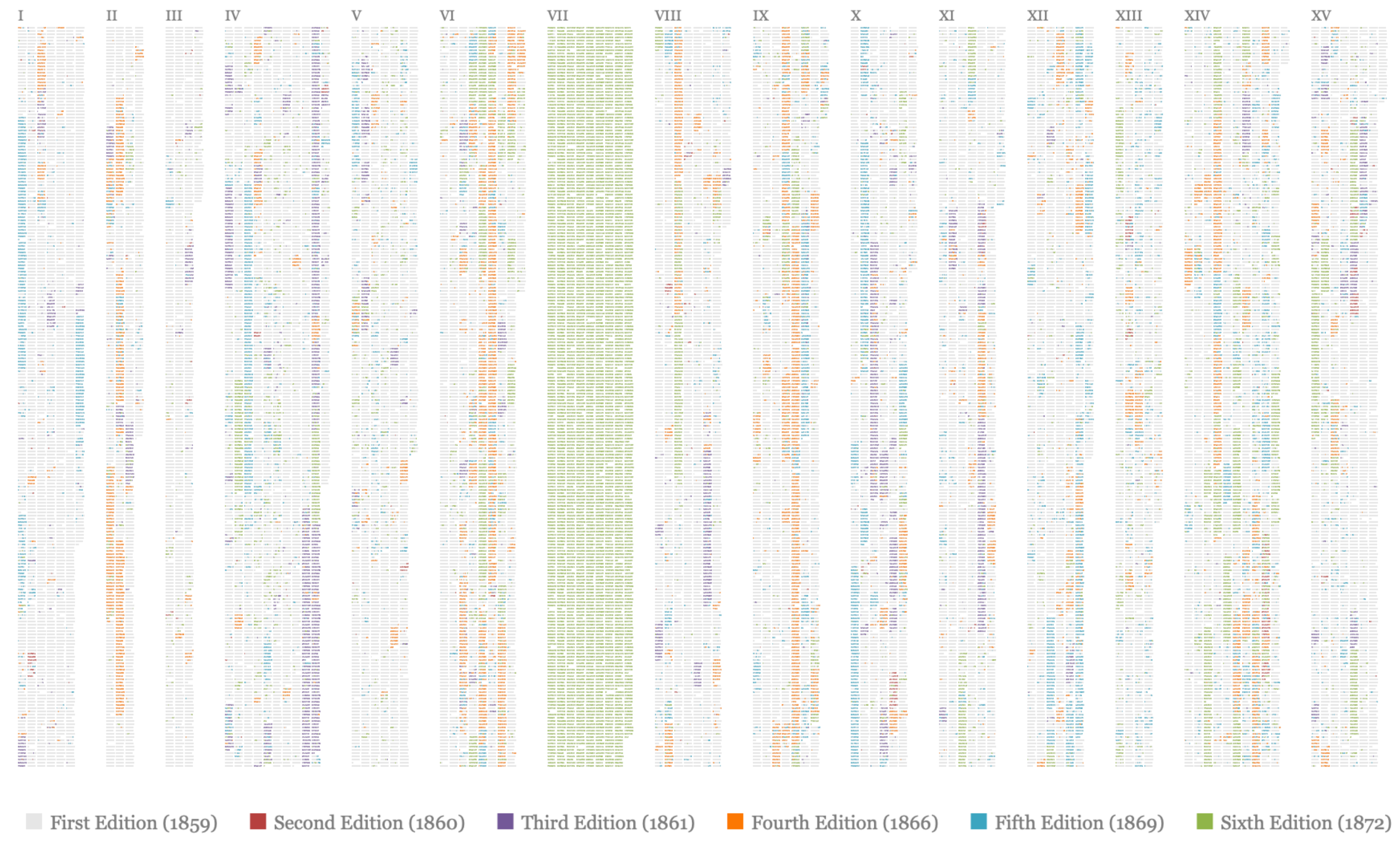

The preservation of Favoured Traces, Ben Fry (2009)

https:/fathom.info/traces/ 


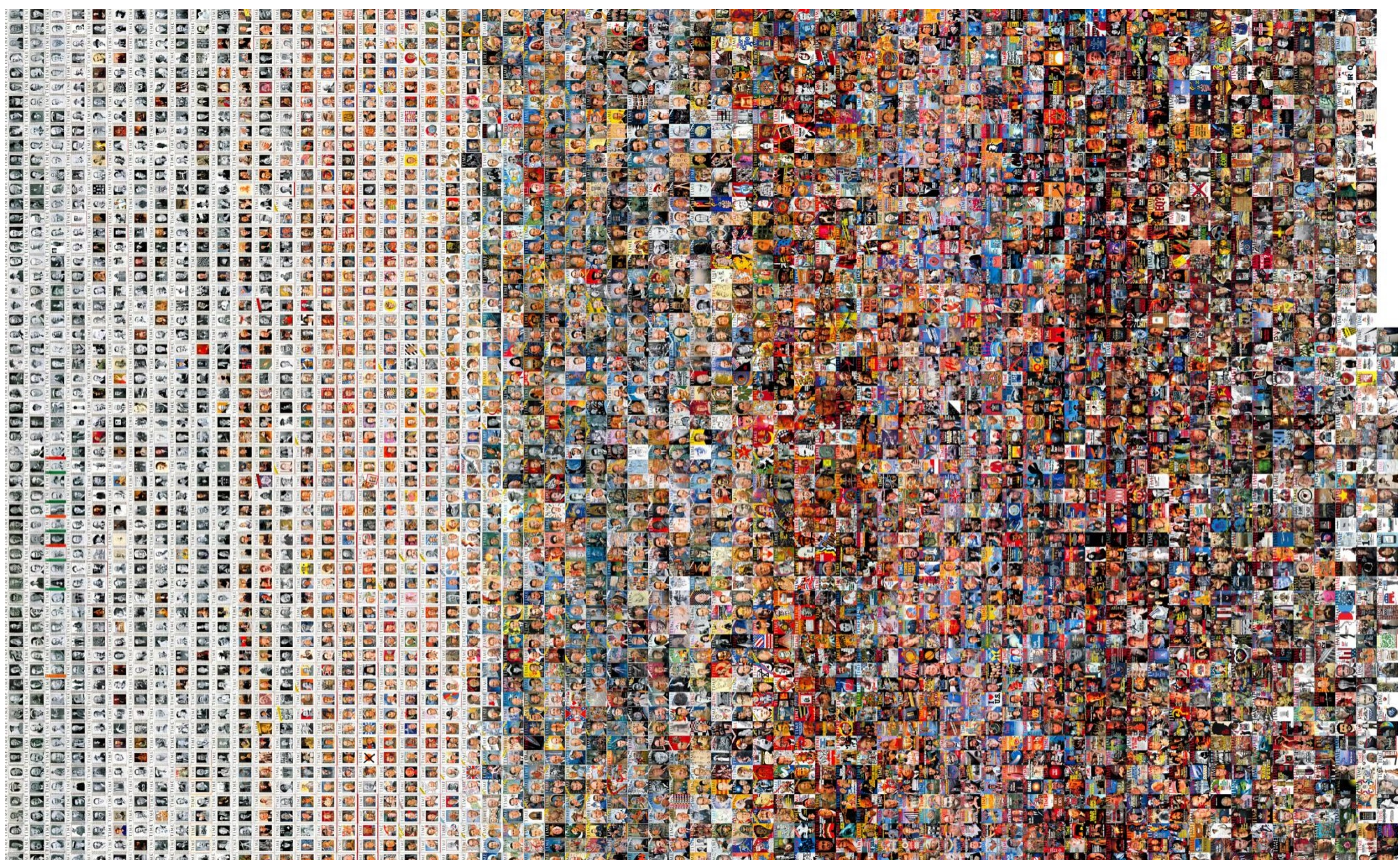

Timeline, Lev Manovich (2010)

http://manovich.net/index.php/exhibitions/timeline 


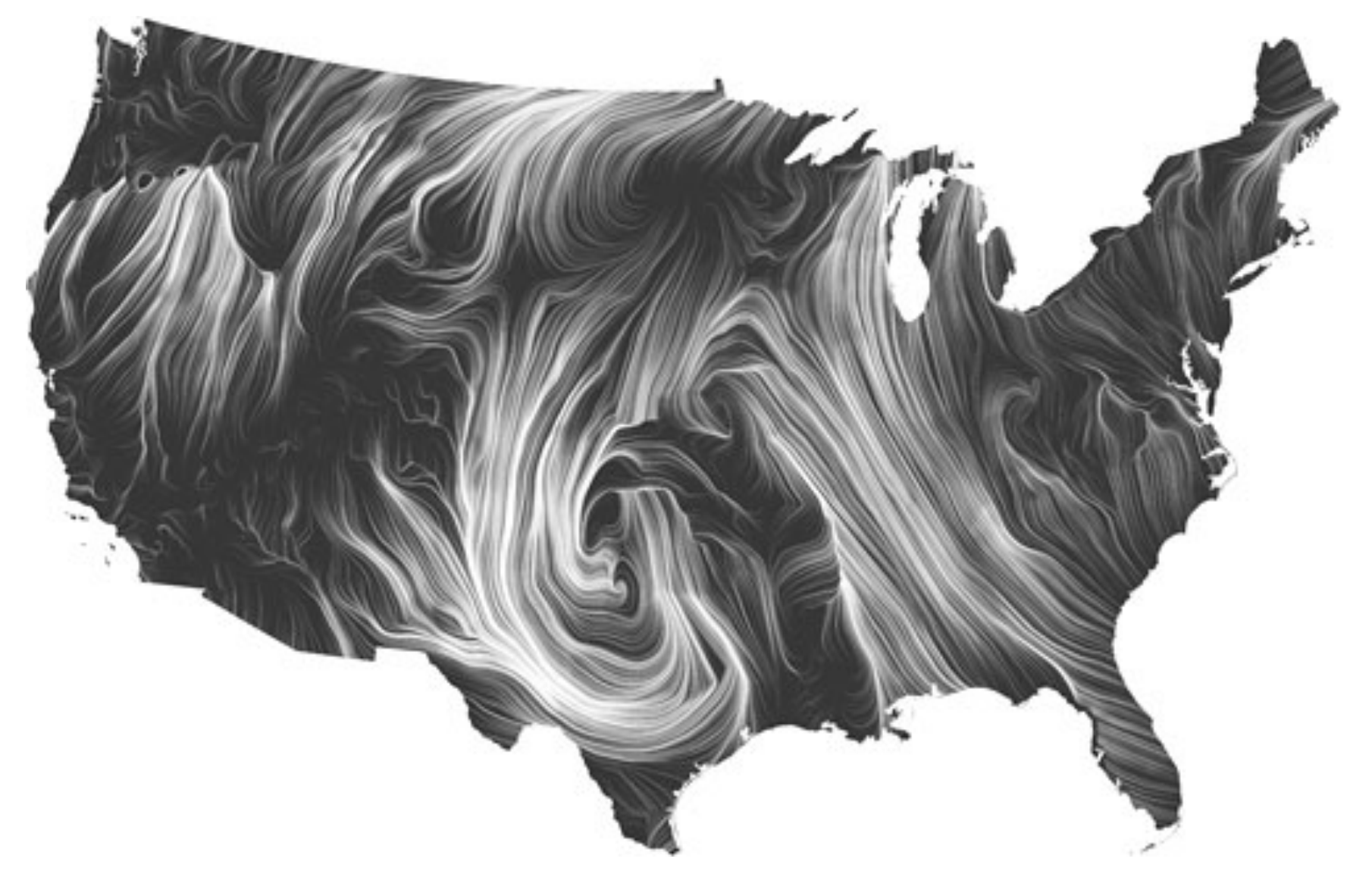

Wind Map, Fernanda Viégas (2012)

http://www.fernandaviegas.com 


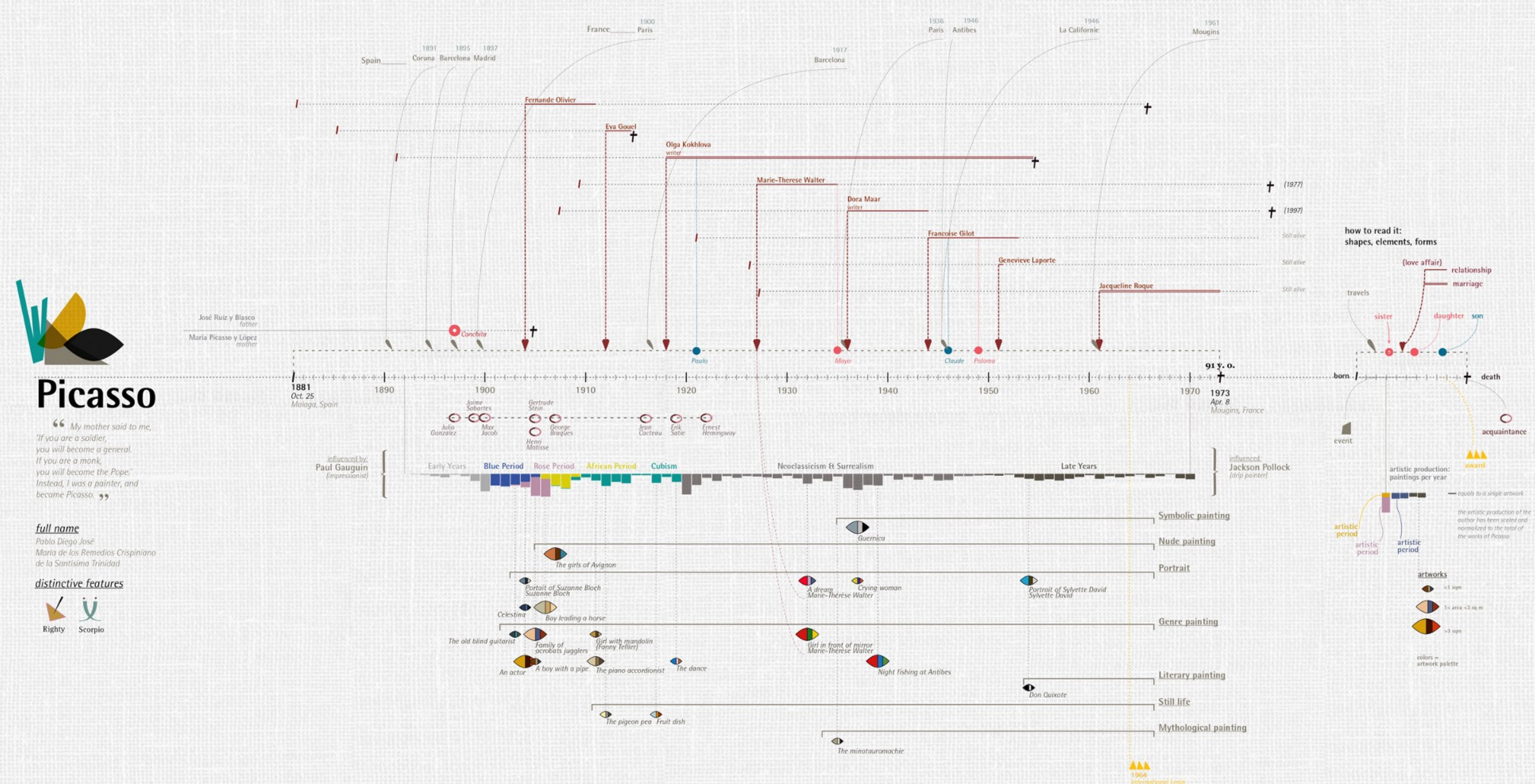

Visualizing Painters' Lives Series, Giorgia Lupi (2013)

https://www.accurat.it 


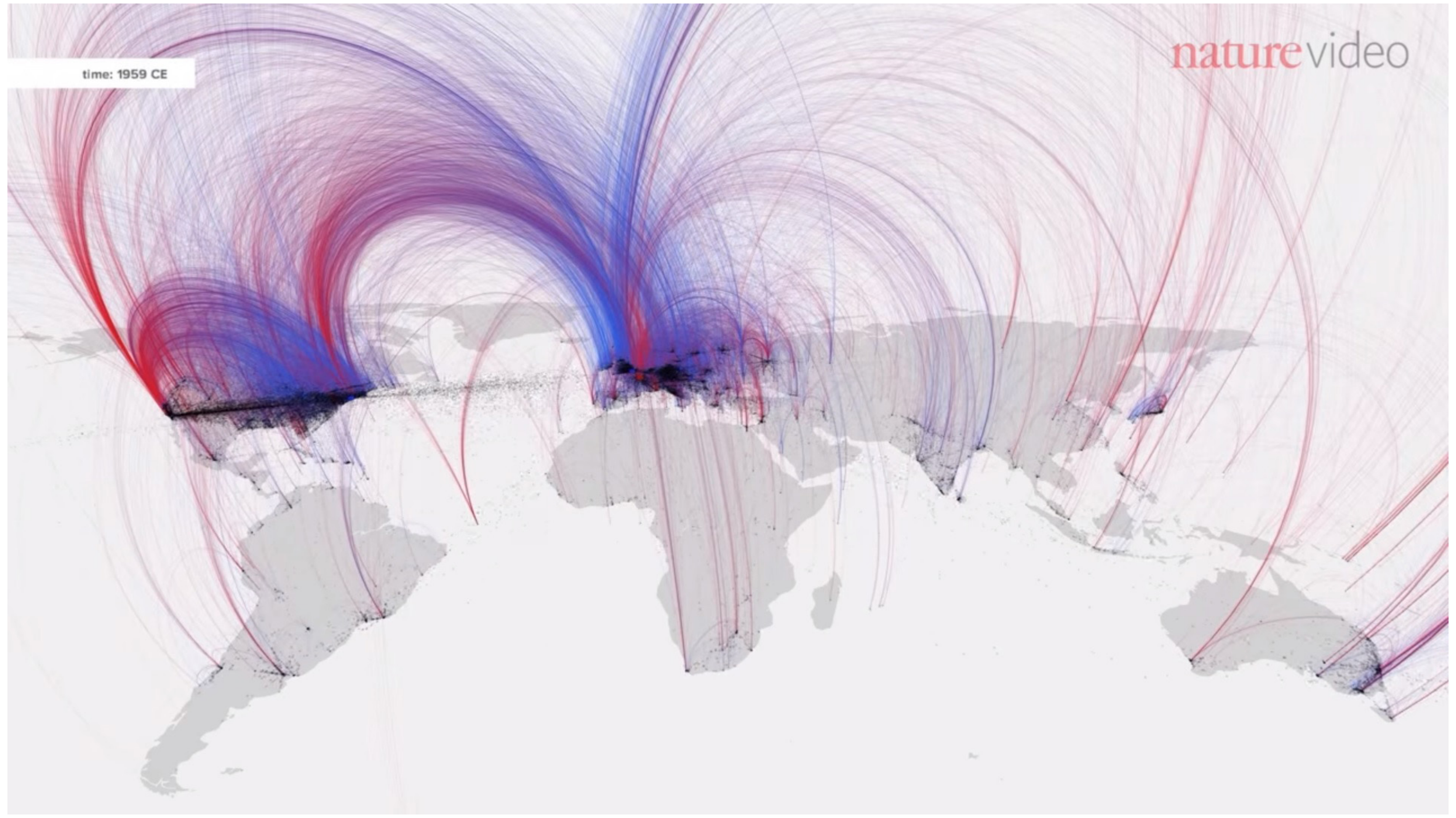

Charting Culture, Mauro Martino (2014)

http://www.mamartino.com/projects/cultsci/ 


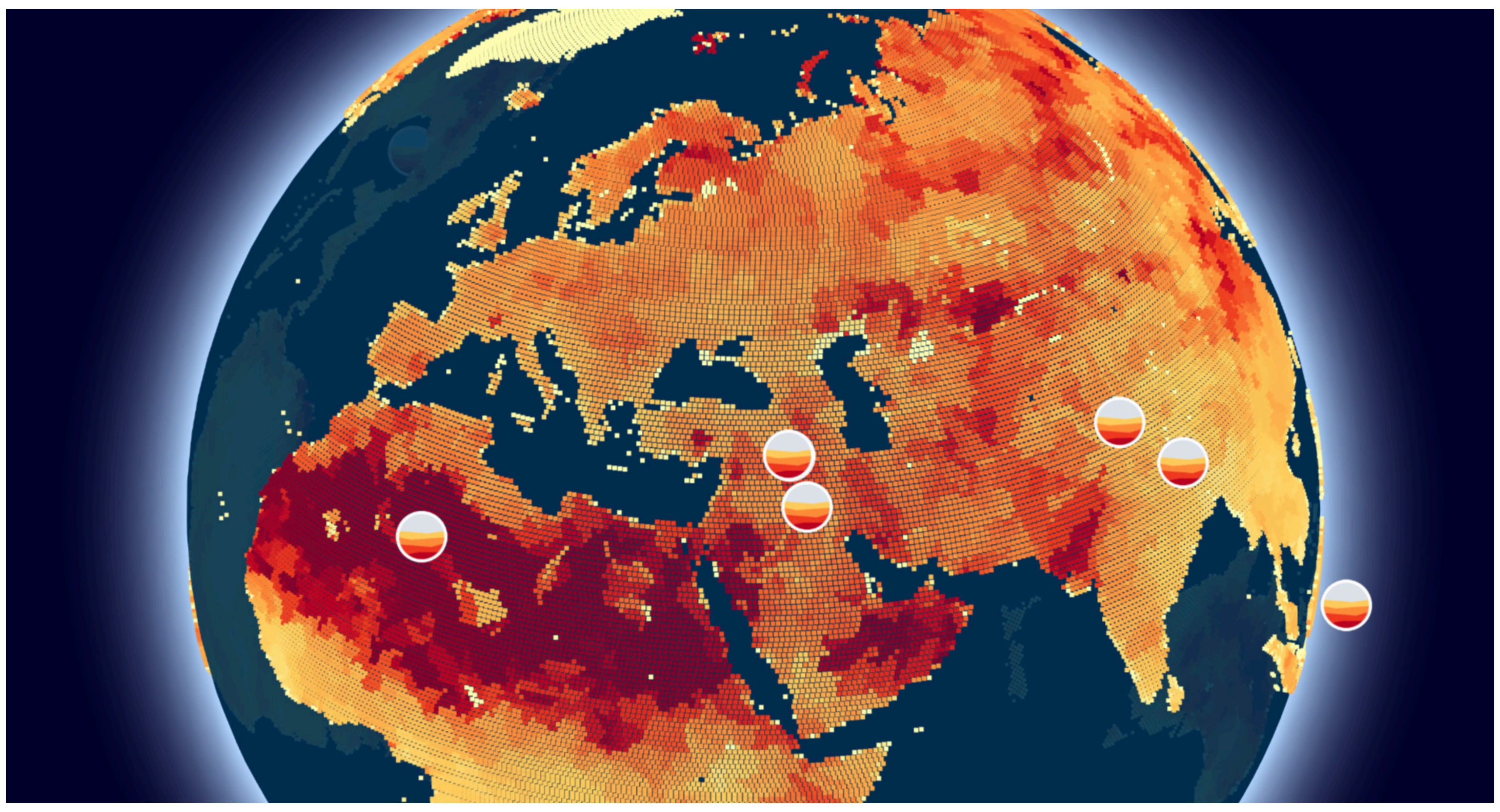

World Water Atlas, Jan Willem Tulp (2016) http://tulpinteractive.com 


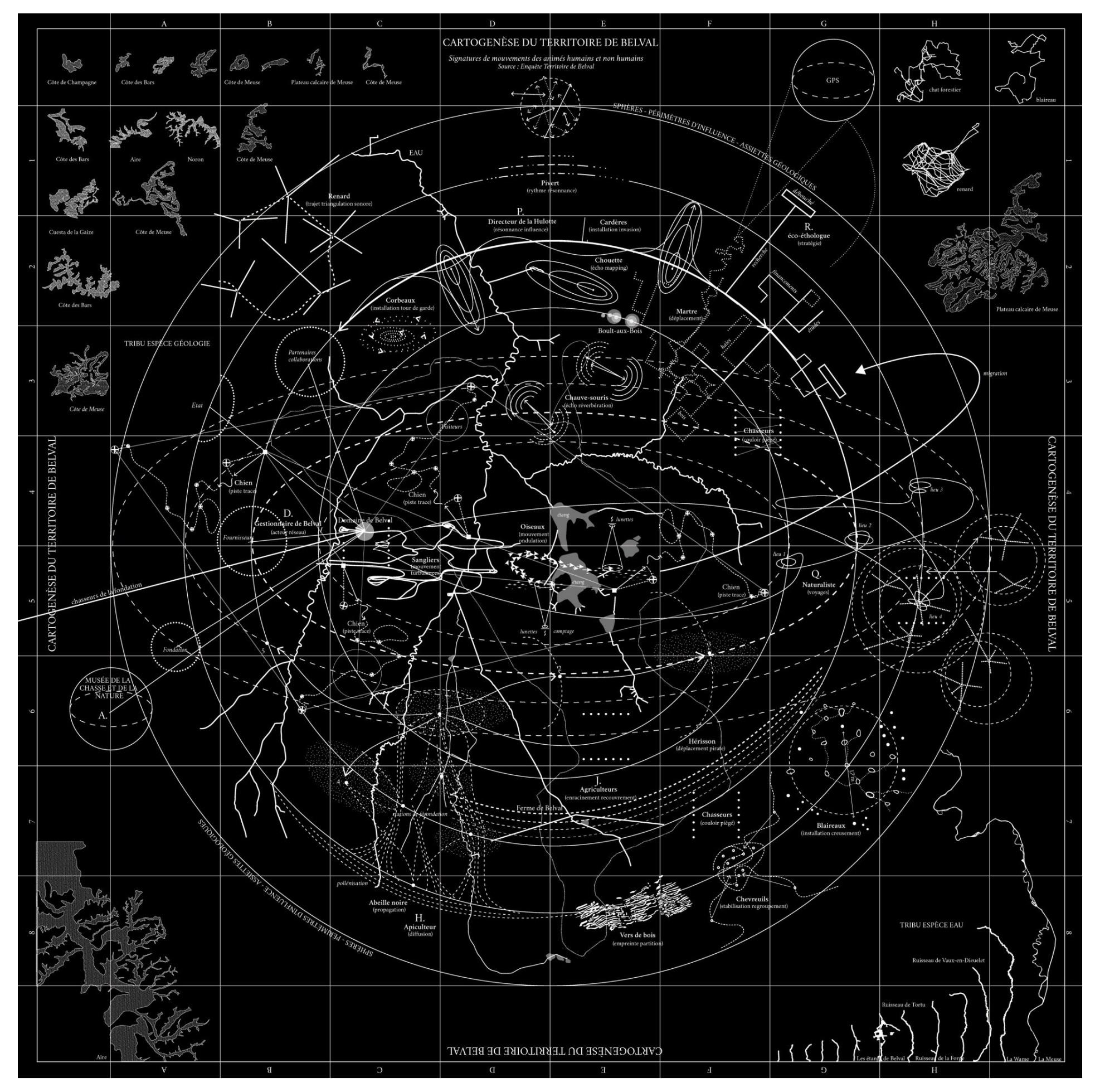

Cartogenese, Alexandra Arenes (2017) https:/vimeo.com/232655044 


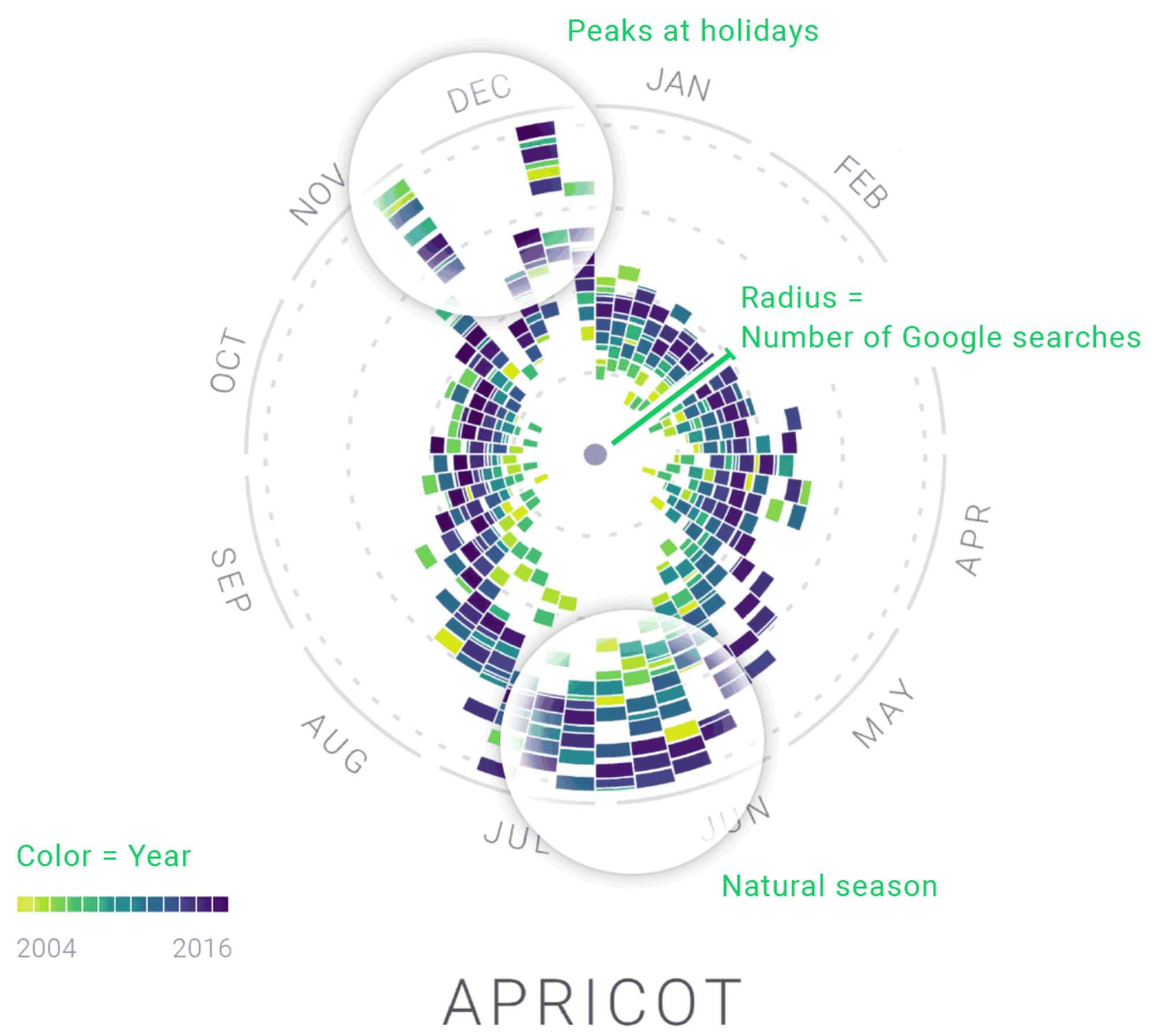

The Rhythm of Food, Moritz Stefaner (2017)

https://truth-and-beauty.net 


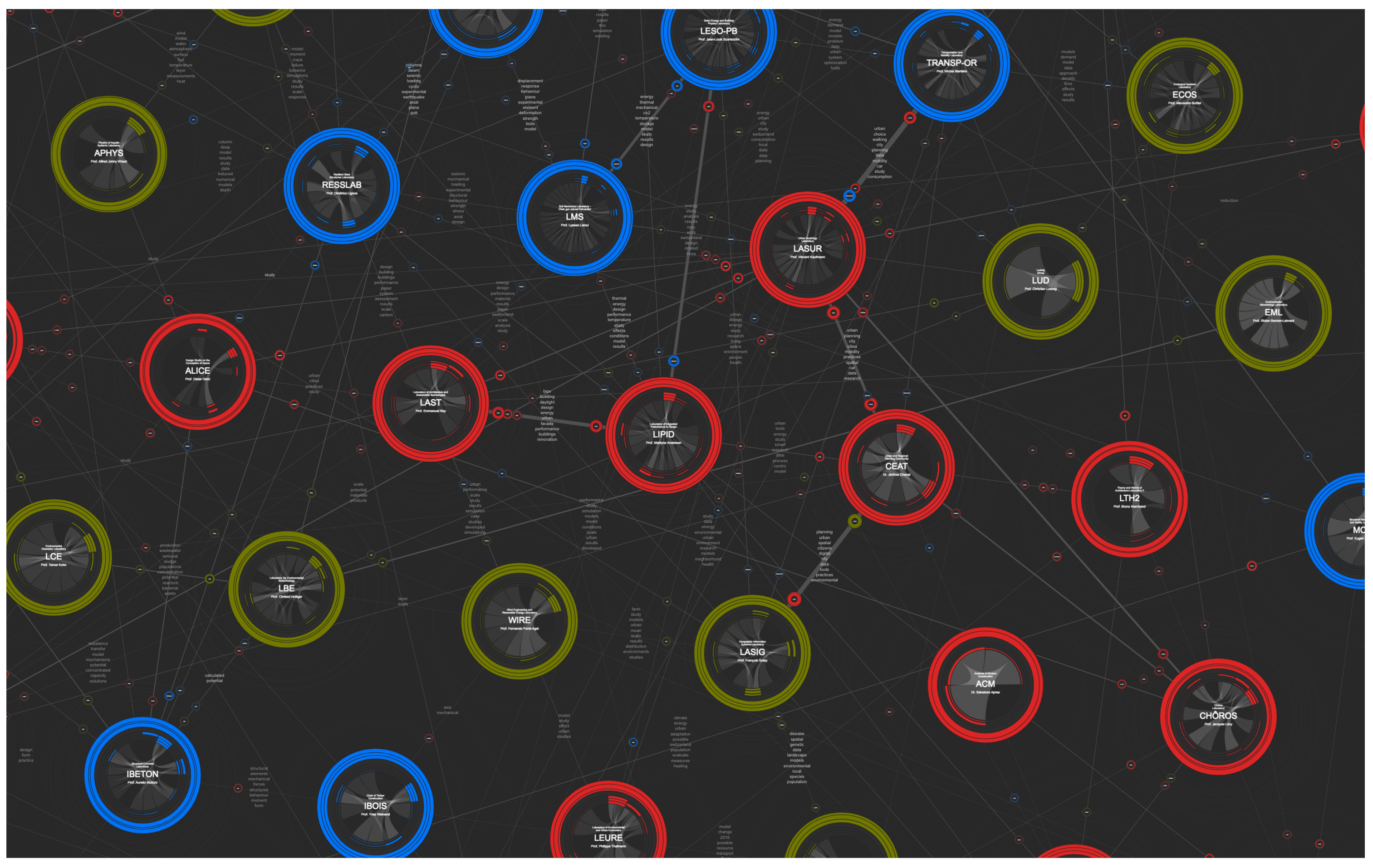

Affinity Map, Dario Rodighiero \& Ogier Maitre (2018) https://affinitymap.epfl.ch 


$$
4 .
$$

\section{To keep in mind}




\section{Structures}

Hierarchical (trees)

Relational (networks)

Temporal (timelines)

Spatial (maps)

Textual (books)

\section{Interpretation}

Association

Differentiation
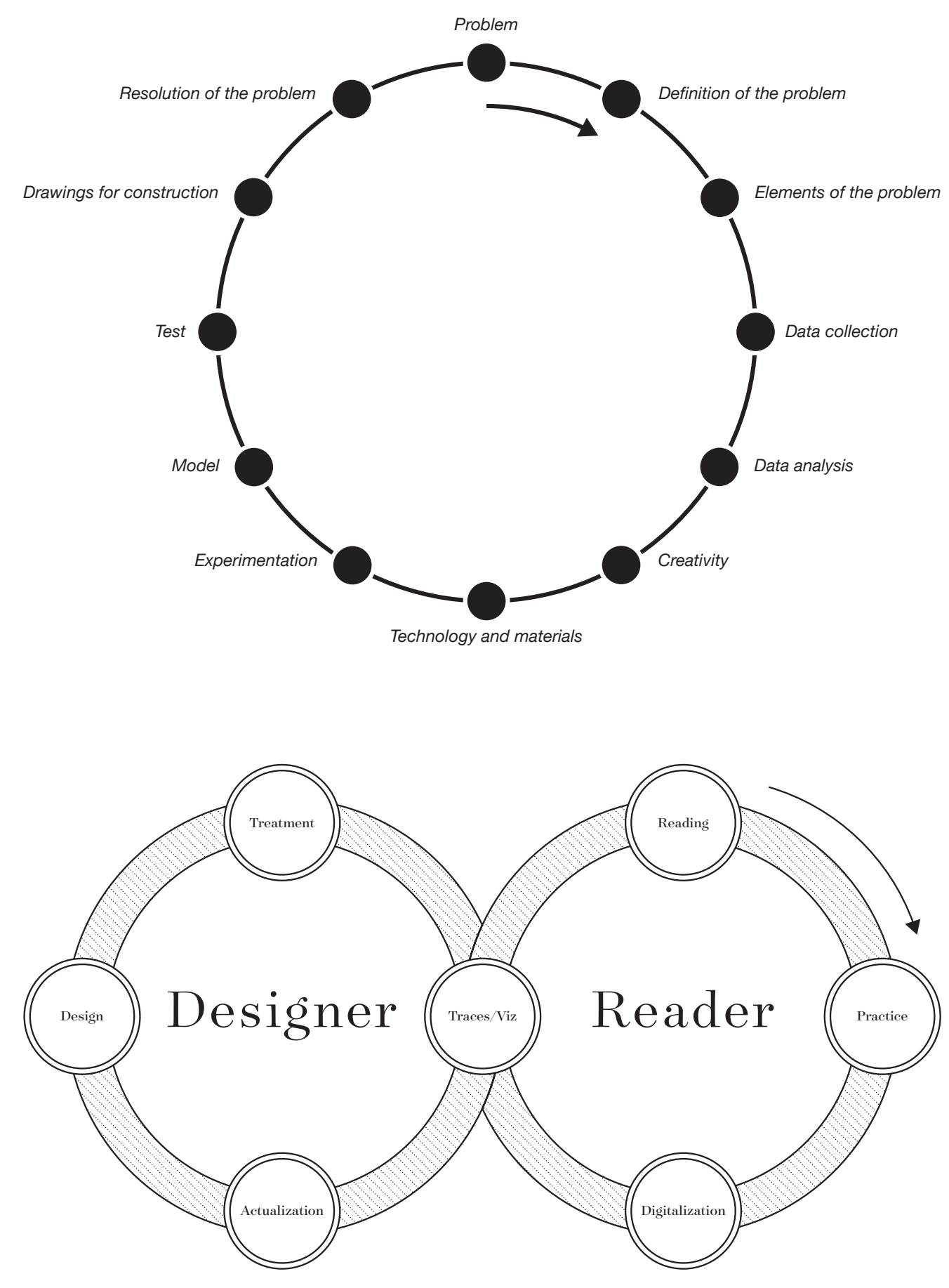


\title{
Thanks
}

\author{
@dariorodighiero \\ dario.rodighiero@epfl.ch \\ https://dariorodighiero.com
}

